\title{
The role of ultrasound-driven microbubble dynamics in drug delivery: from microbubble fundamentals to clinical translation
}

Roovers Silke ${ }^{1}$, Segers Tim², Lajoinie Guillaume ${ }^{2}$, Deprez Joke ${ }^{1}$, Versluis Michel ${ }^{2}$, De Smedt Stefaan C. ${ }^{1}$, Lentacker Ine ${ }^{1}$

\footnotetext{
${ }^{1}$ Laboratory of General Biochemistry and Physical Pharmacy, Ghent Research Group on Nanomedicine, Faculty of Pharmaceutical Sciences, Ghent University, Ottergemsesteenweg 460, Ghent, Belgium

2Physics of Fluids Group, MESA+ Institute for Nanotechnology and Technical Medical (TechMed) Center, University of Twente, P.O. Box 217,
} 7500 AE Enschede, The Netherlands

\section{Abstract}

In the last couple of decades, ultrasound-driven microbubbles have proven excellent candidates for local drug delivery applications. Besides being useful drug carriers, microbubbles have demonstrated the ability to enhance cell and tissue permeability and as a consequence, drug uptake herein. Notwithstanding the large amount of evidence for their therapeutic efficacy, open issues remain. Due to the vast amount of ultrasound- and microbubble-related parameters that can be altered, and the variability in different models, the translation from basic research to (pre-)clinical studies has been hindered. This review aims at connecting the knowledge gained from fundamental microbubble studies to the therapeutic efficacy seen in in vitro and in vivo studies, with an emphasis on a better understanding of the response of a microbubble upon exposure to ultrasound and its interaction with cells and tissues. More specifically, we address the acoustic settings and microbubble-related parameters i.e. bubble size and physico-chemistry of the bubble shell that play a key role in microbubble-cell interactions and in the associated therapeutic outcome. Additionally, new techniques that may provide additional control over the treatment, such as monodisperse microbubble formulations, tunable ultrasound scanners and cavitation detection techniques, are discussed. An in-depth understanding of the aspects presented in this work could eventually lead the way to more efficient and tailored microbubble-assisted ultrasound therapy in the future.

\section{Introduction}

Over the last decades, ultrasound has become an essential tool in diagnostic imaging due to its costeffective, noninvasive and safe nature ${ }^{1,2}$. In ultrasound imaging, an ultrasound beam is transmitted through the body, where it is scattered and reflected at the different interfaces (e.g. fibers, bones...) present $^{3}$. The tissue's distinctive properties result in acoustic impedance mismatches between the tissues leading to different echo intensities, which are displayed in the resulting image as contrast differences. In contrary to other commonly used diagnostic techniques, such as MRI and PET, ultrasound does not need expensive and often stationary equipment, nor the injection of possibly harmful radioactive agents, which makes it an ideal tool for imaging of precarious cases such as 
pregnancy ${ }^{1}$. However, standard (B-mode) ultrasound imaging suffers from poor contrast, which renders the images hard to interpret to an untrained eye.

By serendipity, it was found that injecting saline containing a small dose of tiny gas bubbles tremendously improved the contrast in an ultrasound image ${ }^{4,5}$. Later, the first clinically approved gasfilled microbubbles for diagnostic imaging were introduced. These micron-sized (1-10 micron in diameter) gas bubbles can be intravenously administered and consist of a gaseous core surrounded by a protective shell to extend their lifetime. To further prolong the microbubble lifetime, typically, the microbubbles are filled with high molecular weight gasses with a low aqueous solubility such as perfluorocarbons ${ }^{4,6}$. The microbubble shell can consist of a variety of materials such as proteins, lipids or polymers. The stabilizing shell has been shown to dramatically affect the response of a microbubble to a driving ultrasound pulse and different physical properties result in a different acoustic response ${ }^{7-}$ ${ }^{9}$. Bio-inspired phospholipid-coated shells are of particular interest since they provide a high flexibility while maintaining sufficient resistance to gas diffusion. Consequently, they are the main bubble type encountered both in research and in the clinic, and will therefore be the focus of this review. Apart from the commercial phospholipid-coated microbubble formulations, such as Definity ${ }^{\circledR}$ (Lantheus), Sonazoid ${ }^{\circledast}$ (GE Healthcare) and Sonovue ${ }^{\circledR}$ (Bracco), custom-designed lipid-shelled microbubbles are frequently used in in vivo and in vitro studies since they allow coupling of targeting agents ${ }^{10-13}$, drug carriers $^{10-12,14,15}$ and multimodal imaging agents ${ }^{16-18}$.

The compressibility of the gas core gives microbubbles the ability to contract and expand in response to an ultrasound field. These volumetric oscillations generate harmonic echoes much stronger than the (linear) tissue echoes ${ }^{19}$. This unique feature is at the origin of the "contrast mode" of clinical ultrasound machinery and boosts the microbubble signal to tissue ratio. Since microbubbles are blood pool agents, this feature also enables the visualization and quantification of organ and/or tumor perfusion. Contrast-enhanced ultrasound imaging has become indispensable in the field of echocardiography, and is proving its use in other imaging applications as well. For a more extensive overview of the use of microbubbles in ultrasound imaging, the reader is referred to other reviews ${ }^{20-}$ 23.

Volumetric microbubble oscillations are not only key for contrast-enhanced ultrasonic imaging, but are also crucial for drug delivery applications ${ }^{5,19,24,25}$. Microbubbles oscillating near a cell membrane generate streaming in the surrounding liquid, which puts mechanical stress on the nearby cell. This stress can result in cellular deformation and the creation of small, temporary openings in the cell membrane and in between cells $\mathrm{s}^{26,27}$. These openings can lead to an enhanced uptake of drug molecules in the tissue when drugs are co-administered with microbubbles ${ }^{28}$. Drugs and other therapeutic agents 
can also be directly loaded onto the microbubble shell and ultrasound triggering ensures that they are only released at the desired time and location, which gives the added benefits of preventing their leakage into unwanted tissue or premature degradation of the drug molecule $e^{2,11,29,30}$. This can be especially useful for drugs that have difficulties in reaching the target site in sufficiently high concentrations without inducing side effects, such as chemotherapeutic and anti-inflammatory drugs or in case of easily degradable drugs, such as gene and protein therapeutics. In this way, contrast agent microbubbles become theranostic tools that enable disease monitoring, drug carrier visualization and local drug delivery simultaneously, while strongly enhancing drug uptake into the target tissue.

With this review we aim to connect the knowledge gained from fundamental microbubble studies with a focus on ultrasound-driven microbubble behavior and cellular interactions, to the therapeutic efficacy seen in in vitro and in vivo studies. In other words, we aim to connect the dots between ultrasound parameters and microbubble characteristics used in both the fundamental and clinical fields and provide a comprehensive account of the current knowledge. An in-depth biophysical understanding of microbubble-tissue interactions should ultimately lead to a more predictable, more controlled, and more efficient translation to the (pre)-clinical field.

Part 1: Acoustic microbubble response and its importance for ultrasound-triggered drug delivery

Understanding the response of a microbubble upon exposure to ultrasound is an essential prerequisite to an effective microbubble-assisted ultrasound therapy. Therefore, a vast amount of work has focused on elucidating the mechanical response of a microbubble to ultrasound in terms of the acoustic parameters on the one hand, and microbubble characteristics, i.e. bubble size and physico-chemistry of the bubble shell, on the other hand. In this part we describe what determines the dynamics of a microbubble in response to an ultrasound driving pulse and summarize which aspects are relevant for drug delivery.

\section{The characteristics of ultrasound-driven microbubble oscillations}

As already mentioned, microbubbles will respond to an incoming ultrasound wave through volumetric oscillations. The amplitude of these oscillations highly depends on the ultrasound frequency, pressure, and pulse duration and it is strongly influenced by the microbubble shell and microbubble size. The dynamics of a free microbubble in response to an ultrasonic field, are theoretically described by the Rayleigh-Plesset equation ${ }^{5,6}$. The equation is widely used since it can accurately represent the different microbubble oscillation regimes mentioned below. It can be modified to account for the viscoelastic coating $^{31-34}$, the presence of a nearby substrate ${ }^{35}$ and non-spherical microbubble oscillations ${ }^{36}$. The Rayleigh-Plesset equation is a non-linear differential equation and for any driving pressure, it can be 
solved to give the radius of a microbubble as a function of time $e^{6,37}$. To gain insight in microbubble resonance behavior, it can be linearized. Assuming a sinusoidal acoustic driving pulse, small amplitudes of oscillation with respect to the equilibrium radius, i.e. $R=R_{0}(1+x)$ where $x(t) \ll 1$, and by neglecting higher order terms, linearization of the Rayleigh-Plesset equation leads to the classic equation for a driven mass-spring system, in which the gas can be represented by the spring while the surrounding liquid can be regarded as the mass:

$$
\ddot{x}+2 z \omega_{0} \dot{x}+\omega_{0}^{2} x=\frac{p_{A}}{\rho R_{0}} \sin \omega t
$$

With $x$ a small variation in the microbubble radius $\mathrm{R}, \dot{x}$ and $\ddot{x}$ the first and second time derivative of $x$ respectively, $z$ the damping of the system, $\omega_{0}$ the bubble oscillation eigenfrequency, $p_{A}$ the driving pressure amplitude, $\rho$ the liquid density, $R_{0}$ the equilibrium bubble radius and $\omega$ the angular ultrasound frequency.

A key characteristic of such a mass-spring system is that it contains a resonance frequency $f_{0}=$ $\omega_{0} / 2 \pi \sqrt{1-z^{2}}$, where the oscillation amplitude will be maximal. Simply put, a gas bubble will oscillate in response to an ultrasound pulse, due to two phenomena that push the liquid-gas interface in opposite directions: the resistance against compression of the gas inside the microbubble on the one hand, and the inertia of the liquid on the other hand ${ }^{25,38}$. This competition corresponds to a specific timescale that, at a certain frequency, synchronizes with the ultrasound, resulting in a maximum radial response. This frequency is called the eigenfrequency of the microbubble. However, the microbubble system experiences damping resulting from a loss of energy due to viscous dissipation in the surrounding fluid, re-radiation of sound and thermal losses $s^{6}$. Owing to this damping $(z)$, the resonance frequency is slightly reduced (usually around 1 to $2 \%$ ) compared to the eigenfrequency of the system. The damping also defines the range of frequencies over which the bubble will have a measurable response, which is typically $1 \mathrm{MHz}$ around the resonant frequency. As a result, matching the incoming ultrasound frequency to the resonance frequency of the microbubbles as closely as possible, will be crucial to maximize the acoustic response $\mathrm{e}^{5,19,24}$, as evident in figure $1 \mathrm{~A}$.

$\omega_{0}$ can be defined as:

$$
\omega_{0}=\sqrt{\frac{3 \kappa P_{0}}{\rho R_{0}^{2}}}
$$

with $\kappa$ the polytropic exponent of microbubble filling gas, and $P_{0}$ the ambient pressure. This shows that the resonance frequency is directly related to the microbubble size and therefore, the amplitude of microbubble oscillation is governed by the coupling between the incoming ultrasound frequency and the size of the microbubble. Equation 2 can be simplified by filling in the constants ( $\kappa=1.1$ for 
heavy gasses, $\rho=1000 \mathrm{~kg} / \mathrm{m}^{3}, P_{0}=100 \mathrm{kPa}$ ) to yield equation 3, derived by Minnaert ${ }^{39}$ for uncoated bubbles, namely

$$
f_{0} * R_{0}=3.3 \mu \mathrm{m} * \mathrm{MHz}
$$

where $f_{0}$ is the resonance frequency and $R_{0}$ is the radius of the microbubble at rest ${ }^{5,38}$. Even though equation 3 describes uncoated gas bubbles and thus disregards coating effects and surface tension, it has proven to be a good estimate for the resonance frequency contrast microbubbles ${ }^{37}$.

Yet, typical microbubble ultrasound contrast agents have a broad size distribution with bubble diameters ranging from 0.5 to $10 \mu \mathrm{m}$. Therefore, only a small fraction of the microbubbles will resonate at the acoustic driving frequency and subsequently, only a small fraction of the total bubble population is driven into (maximum) oscillation. To obtain a maximal response, the incident wave should match the resonance frequency of the majority of microbubbles in the population, i.e. the peak in the size distribution. While imaging pulses remain rather broadband ( 70\%) to allow for a good resolution, this matching becomes even more critical in drug delivery application where longer bursts (thus narrower bandwidth) are used to allow the bubble to exert a measurable effect on the target tissue. Another approach to maximize the response of a polydisperse suspension is to use frequency sweeps, also called chirps, where a range of frequencies is used within the same incident wave in order to drive all microbubbles within the population into oscillation ${ }^{5}$. Alternatively, efforts have been made to either sort polydisperse microbubbles according to $\operatorname{size}^{40-44}$, or to produce a monodisperse bubble population using microfluidic flow-focusing techniques ${ }^{45-47}$.

Microbubbles subjected to an ultrasound field can change equilibrium size and therefore resonance behavior as well. Gas leakage out of the bubble through diffusion resulting in bubble shrinkage, can occur due to the changing permeability of the bubble shell during oscillations ${ }^{48}$. In contrast, rectified diffusion can promote bubble growth due to a net influx of gas into the microbubble as a result of both a larger surface area allowing for gas exchange during bubble expansion than during compression and a lower pressure in the expanded bubble, which temporarily decreases the saturation concentration at the bubble surface, thereby accelerating gas intake ${ }^{49}$. As a result, the resonance frequency can change over time. Furthermore, bubbles can cluster due to acoustic radiation forces (which are discussed in more detail later in this work). The resonance frequency of the bubble cluster is different from the single bubbles and will attain an effective resonance frequency of a large bubble with the total gas volume of the bubble cluster ${ }^{5,50}$. Even so, resonance is not based on bubble size alone. Other factors such as physico-chemical properties of the lipid coating and the presence of nearby structures can alter microbubble resonance as well, although the former is mostly important at low driving 
pressure amplitudes $(<50 \mathrm{kPa}$, figure $1 \mathrm{~B})$, which are typically less relevant for drug delivery applications.
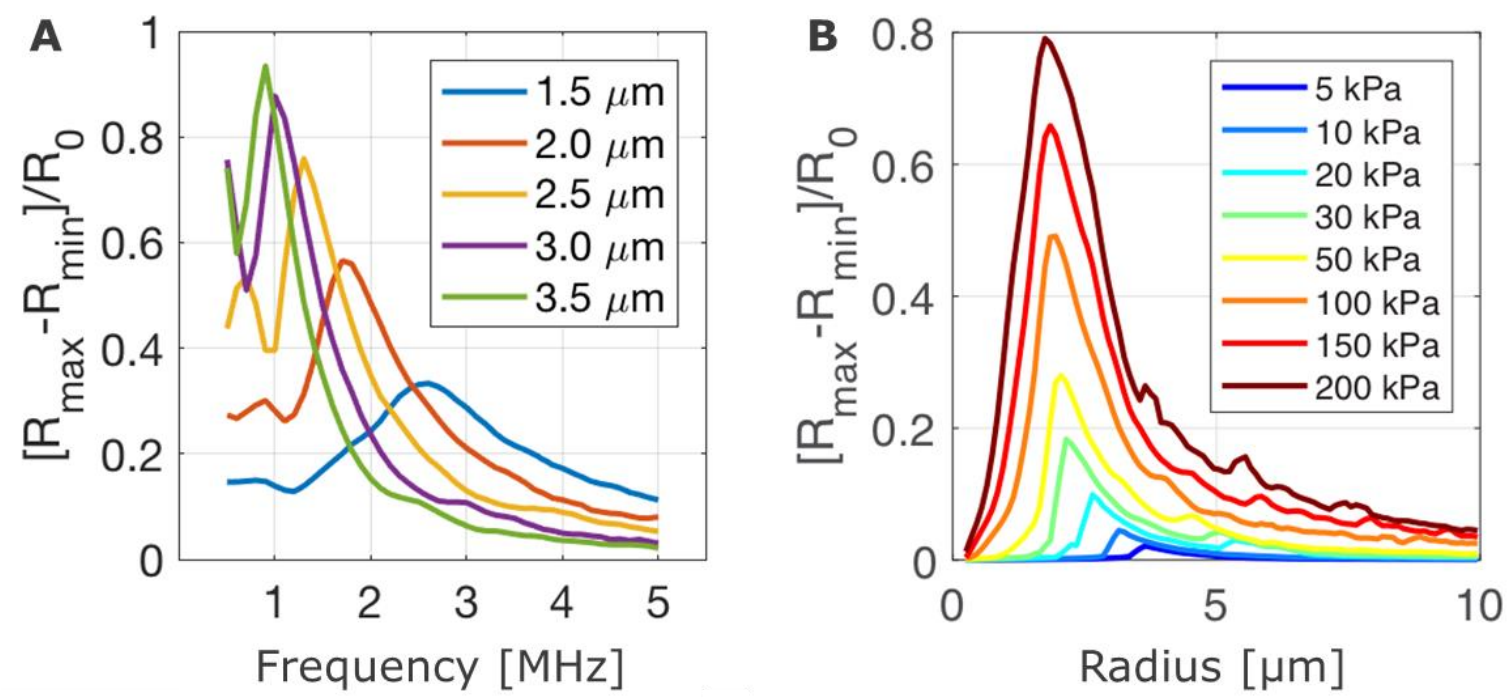

Figure 1: Relative oscillation amplitude of a microbubble with a shell stiffness of $2.5 \mathrm{~N} / \mathrm{m}$, shell viscosity of $6^{*} 10^{-9} \mathrm{~kg} / \mathrm{s}$ and an initial surface tension of $0.02 \mathrm{~N} / \mathrm{m}$, as derived from the non-linearized Rayleigh-Plesset equation. (A) Varying frequency and microbubble size, at a constant pressure of $\mathbf{1 0 0} \mathrm{kPa}$. Microbubbles of different sizes display their maximum amplitude of oscillation at different frequencies of insonation, corresponding to their resonance frequency. The maximum oscillation amplitude decreases for smaller microbubbles due to an increase in damping. (B) Varying microbubble size and acoustic pressure, at a constant ultrasound frequency of $\mathbf{2} \mathbf{~ M H z}$. The maximum amplitude of oscillation is obtained when the frequency of the incoming ultrasound wave matches the resonance frequency of the microbubble, which is dependent on its size and shell characteristics. When the pressure becomes larger than $50 \mathrm{kPa}$, the radius of maximal response remains the same, because the resonance frequency is no longer influenced by shell characteristics.

The lipid coating surrounding the microbubble, while necessary to lower the microbubble's surface tension and prevent gas dissolution in order to extend its lifetime under ultrasound exposure, also hinders the bubble's ability to oscillate to a certain extent. Even though lipid coatings are more flexible than other types of coatings such as polymer coatings, they still add a substantial stiffness and viscosity to the bubble ${ }^{51}$. Practically, for acoustic driving pressures $<50 \mathrm{kPa}$, the presence of a stiff elastic shell increases the microbubble's resonance frequency by a factor 2 to $4^{8}$. This effect becomes negligible for the pressure ranges typically employed in imaging and drug delivery (>50 $\mathrm{kPa})$, since the shell can rupture at large amplitude oscillations. Consequently, the bubble can recover its free gas bubble surface tension and is no longer governed by the microbubble shell. Thus, for driving pressures exceeding $50 \mathrm{kPa}$, the resonance frequency of a coated microbubble can be accurately estimated using the Minnaert equation (Eq.2). The viscosity of the shell does not typically affect resonance frequency but dampens the microbubble oscillations significantly, since it dissipates energy through intermolecular friction between the lipid molecules. Since damping increases with increasing shell viscosity, changing the composition of the shell can have a significant effect on the microbubble 
oscillation amplitude ${ }^{19,52}$. Hence, even bubbles that are the same size and consist of the same type and ratio of shell constituents can have significant variations in their acoustic response $\mathrm{e}^{53-55}$.

\section{Microbubble oscillation regimes at varying driving pressures}

Various oscillation regimes can be distinguished based on the microbubble oscillation amplitude: (i) linear spherical oscillations such as those described by Eq. 1, (ii) non-linear and/or non-spherical oscillations and (iii) inertial cavitation characterized by violent inertial collapses, bubble fragmentation, and the emission of broadband noise ${ }^{5,19}$. Regimes (i) and (ii) are also termed stable cavitation. It is important to keep in mind that the pressures mentioned below only apply to a microbubble driven at its resonance frequency.

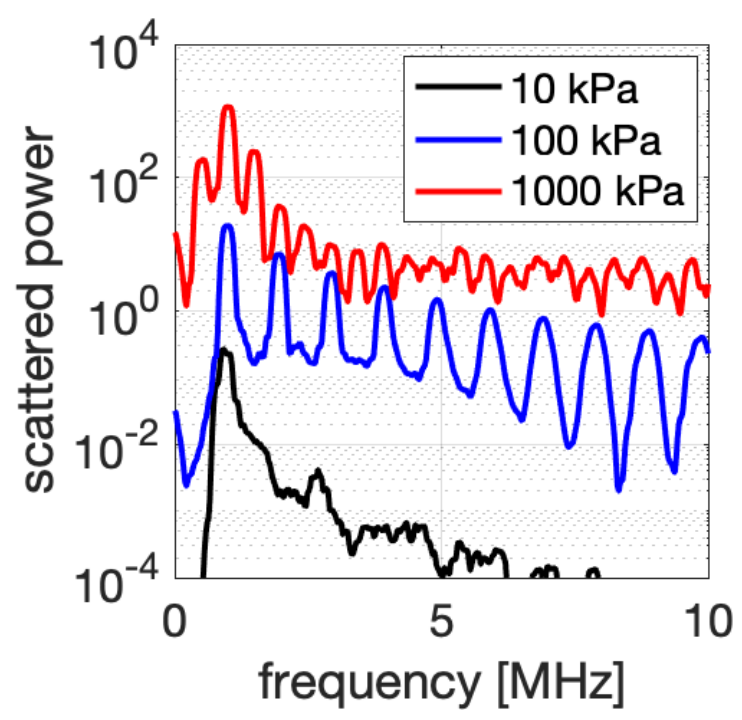

Figure 2: The frequency spectrum of echoes arising from a $3 \mu \mathrm{m}$ microbubble (shell stiffness $2.5 \mathrm{~N} / \mathrm{m}$, shell viscosity $6^{*} 10^{-9}$ $\mathrm{kg} / \mathrm{s}$ and initial surface tension $0.02 \mathrm{~N} / \mathrm{m}$ ) cavitating at $10 \mathrm{kPa}$, at $100 \mathrm{kPa}$ and at $1000 \mathrm{kPa}$ under ultrasound of $1 \mathrm{MHz}$, as simulated using the Rayleigh-Plesset equation. The spectrum at $10 \mathrm{kPa}$ contains mostly the fundamental driving frequency, while the spectrum of $100 \mathrm{kPa}$ shows signs of nonlinear behavior with defined peaks at the harmonic frequencies. Both are examples of stable cavitation. Finally at $1000 \mathrm{kPa}$, the signal increases at the frequencies between the fundamentals, indicative of broadband inertial behavior.

At very low excitation pressures $(<15 \mathrm{kPa})$, microbubbles will oscillate along with the frequency of the incident wave and show stable, symmetrical and relatively small radial oscillations, governed by the microbubble coating ${ }^{5,19}$. Under these low excitation pressures, the echo that is re-radiated by the oscillating microbubble mainly contains the fundamental driving frequency, i.e. the frequency of the incoming ultrasound wave (figure 2, black curve).

Increasing the excitation pressure above $15 \mathrm{kPa}$ will increase the amplitude of the radial oscillations, at which point a threshold for non-linear and/or non-spherical microbubble oscillations is achieved. Nonlinearity is caused by the fact that the expansion phase and compression phase of the microbubble oscillations are no longer equal ${ }^{8,56,57}$, while both coating effects (i.e. buckling, stretching and breaking ${ }^{48,58,59}$; however only relevant for pressures $<50 \mathrm{kPa}$ ) and intrinsic gas core behavior (i.e. 
surface modes ${ }^{60,61}$ ) can lead to a non-spherical response. In addition, the proximity of a rigid wall in studies where bubble dynamics are investigated against a substrate, can be a secondary source of nonlinear and non-spherical effects ${ }^{36,62-66}$. This nonlinear and/or non-spherical response is evident from the microbubble echo that now not only contains the fundamental driving frequency but also harmonics and subharmonics thereof (figure 2, blue curve). Non-linear and non-spherical behavior can lead to microstreaming patterns around the microbubbles since the fluid around the microbubbles will be affected in an inhomogeneous, asymmetric way ${ }^{31,38}$. Microstreaming can transport molecules to and away from the microbubbles, such as drugs for instance, and can generate shear stresses on structures around the microbubbles ${ }^{57,67}$. The ability to induce mechanical stress is particularly interesting for therapeutic applications since it can open cellular tight junctions ${ }^{68}$ and create pores in cell membranes ${ }^{69}$. Since the echoes from the microbubbles contain harmonics and subharmonics of the driving ultrasound frequency under this regime, it is especially useful for clinical ultrasound imaging as it allows the discrimination between the signal from the microbubbles and that scattered from surrounding tissues. The harmonics and subharmonics are interesting to discern stable from inertial cavitation during drug delivery experiments as well.

If the excitation pressure increases even further (approx. $>300 \mathrm{kPa}$ ), the microbubble behavior becomes chaotic and the oscillation amplitude grows so large during rarefaction that the inertia of the surrounding fluid will eventually overcome the pressure inside the gas bubble, followed by bubble collapse and/or by fragmentation into smaller bubbles ${ }^{5,19}$. This regime is called inertial cavitation. In terms of therapy, the inertial cavitation regime can cause shock waves and jet formation that can have significant effects on surrounding cells ranging from pores in the cell membrane to cell death ${ }^{66,70}$. The regime no longer elicits well-defined harmonic echo signals but rather broadband noise over a broad range of frequencies ${ }^{19}$ (figure 2, red curve). Since the microbubbles are destroyed, resulting in loss of contrast, and since the violent collapses may cause hemorrhage and tissue necrosis in the surroundings, this regime is less suitable for imaging purposes. In practice, the threshold between inertial and stable cavitation is not well-defined and bubbles start to cavitate inertially over a range of acoustic pressures ranging from roughly 200 to $500 \mathrm{kPa}$.

Since the ultrasound pressure is an important parameter to determine the oscillation regime, it stands to reason that this ultrasound parameter is most often reported in literature. However, as mentioned above, the relation between the acoustic pressure and microbubble dynamics only applies when microbubbles are excited near their resonance frequency. In reality, microbubbles are often driven out of resonance. Therefore, it proves difficult to make clear conclusions and comparisons between the results from different research groups solely based on the acoustic pressures reported. To find a more suitable parameter to compare microbubble behavior, other options could be explored such as the 
relative amplitude of oscillation, $R_{\max }-R_{\min } / R_{0}$, as used by Kooiman et al. ${ }^{71}$, Helfield et al ${ }^{27}$ and Luan et $\mathrm{al}^{72}$. This takes into account the size and coating-related factors as well as the impact of the ultrasound regime. Unfortunately, relative oscillation amplitudes can only be determined with ultra-high-speed imaging systems operating at an imaging frequency that must exceed the ultrasound frequency multiple times $^{73}$ and is therefore not in reach for the majority of research groups working on microbubble-assisted drug delivery. Alternatively, the use of monodisperse microbubbles results in more controlled bubble dynamics due to a homogeneous acoustic response ${ }^{74}$ and therefore, comparing results based on such formulations will potentially be more straightforward.

\section{Microbubble shell shedding to release therapeutic payload of drug-loaded microbubbles}

As microbubbles can be designed to hold various therapeutic payloads on their surface, it is important to consider if, when and how this payload is being released. It was already suggested by Borden et al. ${ }^{48}$ that due to the surface area reduction in the compression phase, excessive shell material could be expelled. This could result in the shedding of the lipid material and therefore the therapeutic payload will be shed as well. Subsequent studies have focused on the requirements for microbubble shell shedding in response to ultrasonic radiation and have found that shedding of the microbubble shell is strongly correlated with a reduction of the surface area leading to an oversaturation of the lipid shell material $^{75-77}$. Later, a relative bubble oscillation amplitude of at least $30 \%$ was observed as the threshold for lipid shedding of DPPC coated microbubbles ${ }^{67,72}$. This could already be achieved with pressures around $200 \mathrm{kPa}$ (figure 3) 72 . Below this shedding threshold, the shell material was not released but rather a rearrangement of the shell constituents and buckling was observed ${ }^{58,72}$.
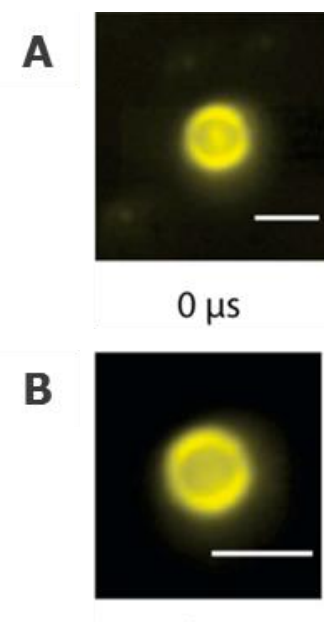

$0 \mu s$

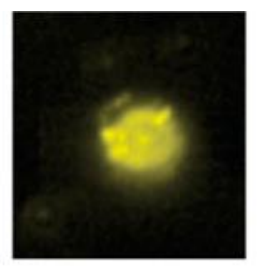

$140 \mu \mathrm{s}$

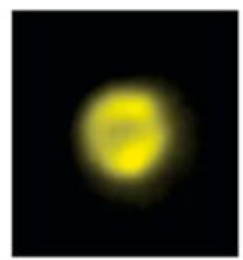

$20 \mu \mathrm{s}$

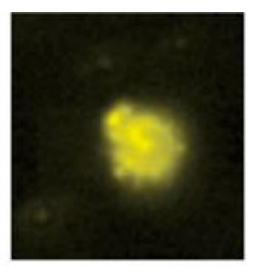

$213 \mu \mathrm{s}$

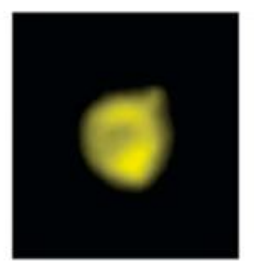

$60 \mu \mathrm{s}$

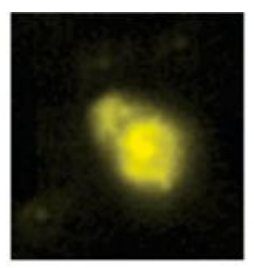

$267 \mu \mathrm{s}$

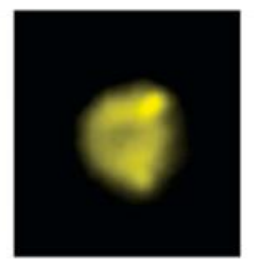

$80 \mu \mathrm{s}$

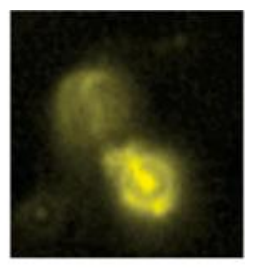

$520 \mu \mathrm{s}$

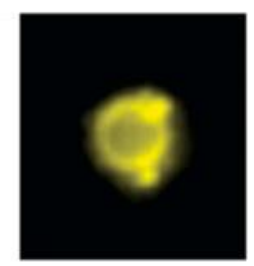

$120 \mu \mathrm{s}$

Figure 3: DPPC-based microbubbles with fluorescent Dil in the shell, to show the shedding events under ultrasound radiation at $1 \mathrm{MHz}$ center frequency. (A) The shell material is released from the microbubble core under the influence of an ultrasound pulse of $170 \mathrm{kPa}$ and 500 cycles, while in (B) no shell shedding could be observed at $255 \mathrm{kPa}, 100$ cycles. Instead shell budding was observed. In both cases the scale bar represents $5 \mu \mathrm{m}$. Reproduced from Luan et al ${ }^{72}$ with permission from Elsevier. 
Shell shedding is again highly dependent on the microbubble shell composition. For example, Borden et al. showed that longer acyl chains resulted in coatings that were more resistant to acoustic dissolution, which in turn lowered their propensity to shedding ${ }^{48}$. This can be explained by the higher cohesiveness (intermolecular forces) and elasticity of these kind of shells. Van Rooij et al. confirmed this by showing that DPPC based-microbubbles are less acoustically stable then their DSPC (C18) variants $^{78}$. Additionally, Kooiman et al. ${ }^{58}$ demonstrated that at a relative vibration amplitude of $30 \%$ was not enough to initiate shell shedding of DSPC bubbles, but rather resulted in enhanced lipid bucking and folding in the surface structure of the bubbles instead. The shedding of the shell components can be affected by loading therapeutic agents onto the surface of the microbubbles too, as had been found by Luan et $\mathrm{al}^{57}$. They saw that a higher pressure was needed for vibration and shedding when nanoparticles were covalently coupled onto the microbubble shell, due to a higher viscosity of the shell, in particular for larger microbubbles ${ }^{51}$. In conclusion, whereas the microbubble shell has little influence on the resonance frequency at the acoustic pressures used for therapy, it may have a dramatic effect on the shedding of therapeutic compounds loaded in or on the microbubble shell through shell damping. Hence, it is important to keep in mind that whenever alterations to the microbubble composition are made, for example to allow coupling of therapeutic agents or targeting agents, careful consideration of the effects on the acoustic response is required as well.

\section{Radiation forces leading to microbubble translation}

Apart from radial oscillations, microbubbles can also respond to an incoming ultrasound wave by translating under the influence of the so-called acoustic radiation forces, or Bjerkness forces $19,24,25,38,79$ (figure 4). These forces arise at any acoustic pressure but their effects become increasingly important with longer acoustic pulses and higher intensities and result in a net time-averaged force directed away from the transducer ${ }^{24,25,38}$. Furthermore, since the microbubbles themselves act as a secondary source of ultrasound waves when cavitating, they will also exert secondary forces onto neighboring bubbles ${ }^{19}$. As a result, microbubbles oscillating in phase in close vicinity of each other will experience a net attractive force (figure 4B). This can result in microbubble aggregation and eventually lead to microbubble coalescence ${ }^{5,19,79,80}$. Primary radiation forces can be useful for molecular imaging purposes as they can be applied to push targeted microbubbles towards ligands lining the vessel wall, in order to stimulate microbubble binding ${ }^{81-83}$. Additionally, these forces can be involved in therapeutic applications since they can be used to push drug-loaded microbubbles towards their target in order to maximize drug delivery ${ }^{84,85}$, as shown in figure $4 A^{86}$. 

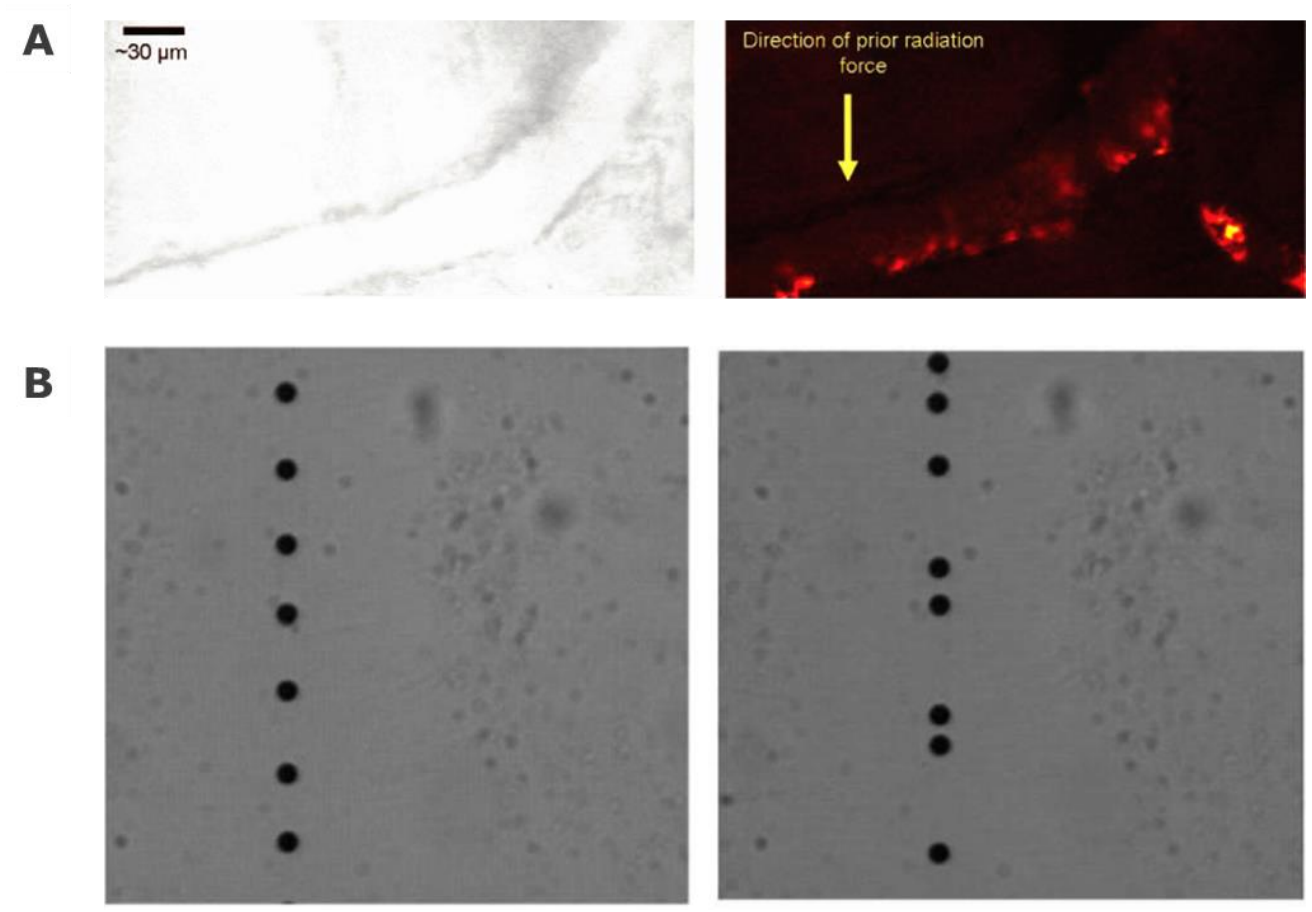

Figure 4: (A) Deposition of fluorescent model drugs attached to microbubble-like acoustically active lipospheres on an ex vivo capillary wall, after a specific acoustic radiation force - fragmentation ultrasound pulse sequence. The primary acoustic radiation force is used to push the microbubble-like vehicles in the direction of the distal wall, where the payload can be deposited using the fragmentation pulse. (B) Secondary radiation forces cause individual air bubbles ( $53 \mu \mathrm{m})$ to move towards each other under a single pulse of $110 \mathrm{kPa}$ when their oscillations are in phase. The same mechanism applies for microbubbles. (C2018 IEEE. Reprinted, with permission, from Shortencarier et al. ${ }^{86}$ and Palanchon et al. ${ }^{87}$.

\section{The presence of nearby structures}

The presence of nearby substrates such as rigid membranes present in in vitro studies and even more compliant viscoelastic boundaries found in vivo, can influence the microbubble oscillation mode and as such acoustic radiation forces and microbubble shell shedding. In terms of microbubble oscillation dynamics, it is known that structures in the vicinity of an oscillating microbubble can affect the resonance frequency and maximum oscillation amplitude ${ }^{4}$. For example, microbubble vibrations were shown to be smaller in case of vascular confinement ${ }^{88}$. Depending on the mechanical properties of the substrate, theoretical studies have shown that the microbubble resonance frequency can either increase or decrease, however this still needs further investigation ${ }^{89,90}$.

Secondly, when a microbubble is oscillating next to a membrane or wall with a higher acoustic impedance than the surrounding medium, this membrane will act as an ultrasound wave reflector and causes the creation of a virtual image bubble oscillating in phase with the real microbubble ${ }^{5,36}$. Due to secondary radiation forces the microbubble will be attracted to this image bubble and will move towards the membrane, as visualized in several in vitro experiments ${ }^{62,66}$. This can affect the outcome of several in vitro drug delivery studies as these are often performed with membrane adherent cells. 
Finally, numerical modeling and experimental studies have been performed to investigate microbubble shell shedding when a microbubble is oscillating asymmetrically in the presence of a nearby membrane ${ }^{36}$. Microbubbles that oscillate near a membrane will create axisymmetric microstreaming patterns since their vibration will be affected by the presence of that membrane ${ }^{31,36,91}$. Upon insonation, oversaturation of the lipids occurs in the shell region near the wall, where the lipids finally detach and are transported away from the membrane via microstreaming, as represented in figure $5^{36}$. Considering this directional shedding can be important in drug delivery studies as it actually transports the drugs away from the cell-carrying membrane. Yet, it remains unclear what the impact on an in vivo situation can be, as these microstreaming patterns will depend on the mechanical properties of the nearby membrane, which differ greatly between in vitro studies and the more compliant walls present in vivo.
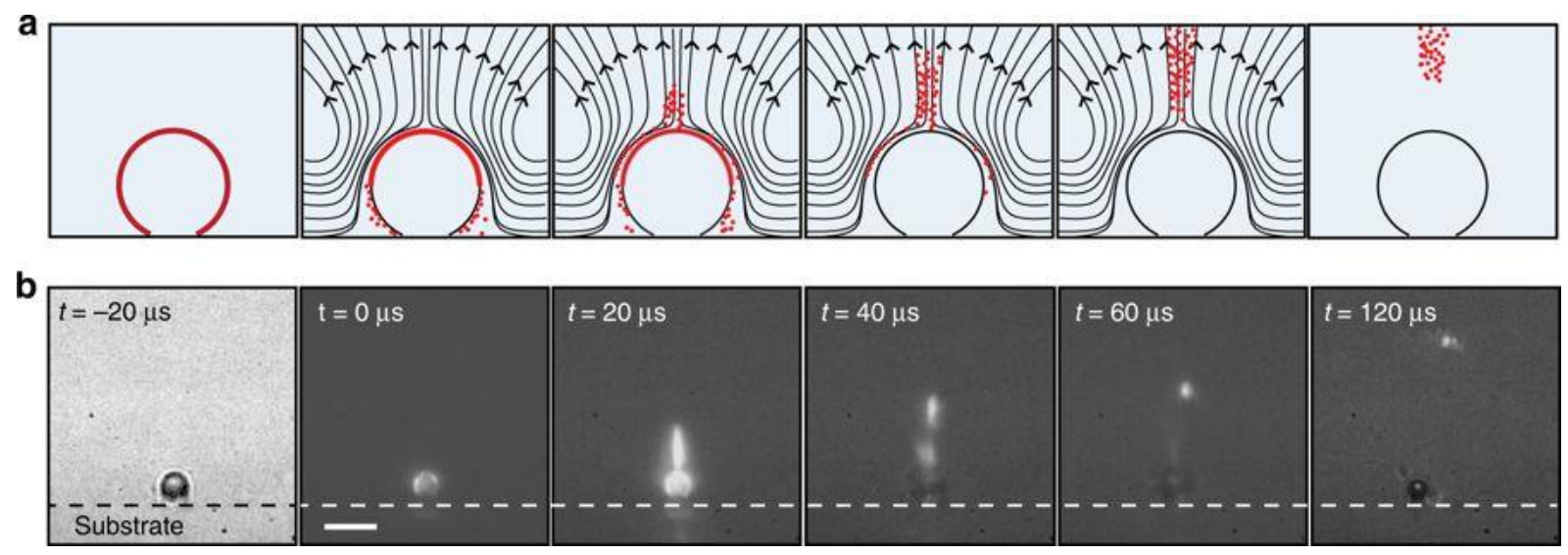

Figure 5: (A) Schematic representation of shell shedding when a microbubble is in close proximity to an acoustically reflective wall. The red dots represent microbubble shell lipids while the black lines show the calculated streamlines. (B) Asymmetrical shell shedding as observed in side-view at a pressure of $331 \mathrm{kPa}$. The scale bar represents $10 \mu \mathrm{m}$. Reproduced from Lajoinie et al. ${ }^{36}$ under the Creative Commons Attribution 4.0 International License: http://creativecommons.org/licenses/by/4.0/.

\section{Part 2: Microbubble-cell interactions to release and deliver drugs to tissue}

\section{Methods used to unravel microbubble-cell interactions}

Studying the microbubble-cell interactions that lead to intracellular delivery of drugs is challenging since they occur on various time scales, length scales and involve numerous physical, chemical and biological parameters ${ }^{4}$. Therefore simultaneous use of multiple techniques is required to obtain a complete overview. Microscopy-based techniques are ideal to manage the multiple time scales since imaging rates can vary from real-time imaging (a few frames per second) allowing evaluation of drug influx and cellular responses ${ }^{92}$; to high speed (150 $000 \mathrm{fps}$ ) imaging which can resolve the release of model drugs from microbubbles and streaming effects ${ }^{72}$; and even to ultrahigh speed imaging (up to 25 million fps) making it possible to resolve the microbubble oscillations during ultrasound exposure ${ }^{58,73}$. Other methods can be used to evaluate the consequences of microbubble-cell interactions during or after ultrasound treatment ${ }^{4}$. For example, the integrity of cell membranes and 
intercellular junctions can be measured using electrical sensing techniques (TMC, TEER) ${ }^{93,94}$. The proteomic and transcriptomic changes in the exposed tissue can be analyzed using western blots ${ }^{95-97}$, ELISA assays ${ }^{97}$ and PCR analysis ${ }^{96,97}$. The effects can be studied in further detail quantitatively using flow cytometry and qualitatively using electron and atomic force microscopy. Nevertheless, owing to this inherent complexity, the interpretation and integration of these different techniques remains a significant challenge.

\section{Mechanisms of ultrasound and microbubble-induced drug delivery at the single-cell level}

The best-known and most-studied microbubble-associated drug uptake mechanism is sonoporation $^{24,26,27,69,98,99}$ (figure 6A). Sonoporation can be defined as the formation of transient openings in the cell membrane induced by microbubble oscillations, through which molecules can passively diffuse in and out of the intracellular space. These pores can range from ten nanometer to several hundred nanometers and even a few micrometers in size ${ }^{24,100,101}$. However, for a cell to remain viable, the pores should close within a reasonable time frame and should therefore be sufficiently small to completely reseal during that time. Studies that have looked into these aspects have found that pores should be smaller than $100 \mu \mathrm{m}^{2}$ and ideally even smaller than $30 \mu \mathrm{m}^{2}$ to reseal quickly and succesfully ${ }^{26}$, while resealing times were reported to be anywhere between a few seconds and 120 seconds $\mathrm{s}^{26,69,94,102}$.

The disruption of the plasma membrane during sonoporation will have several bio-effects since this membrane is crucial in maintaining the balance between the intracellular and extracellular environment, which is indispensable for the normal function of the cell. These bio-effects may be caused by a direct mechanical effect of the microbubble oscillations but can also be triggered by chemical effects caused by oscillating microbubbles. These include the formation of reactive oxygen species that can alter cell membrane integrity and affect ion channels ${ }^{100,103}$. The direct connection between the intracellular and extracellular space during sonoporation allows molecules to leak into the cells, but will also result in the leakage of intracellular constituents. As a consequence, sonoporation can be detected through the influx of otherwise impermeable agents such as propidium iodide $^{69,102}$, as well as through the loss of intracellular agents such as calcein or GFP from stably transfected GFP cell lines ${ }^{104}$. Moreover, it can lead to the influx and outflux of ions, which results in a disruption of the transmembrane potential ${ }^{105,106}$. One ion that has been of particular interest is calcium. Since the calcium concentration is higher in the cell surroundings than in the intracellular environment, sonoporation will lead to an influx of calcium, which can influence several intracellular processes and was found to play an important role in membrane recovery after sonoporation ${ }^{24,107-109}$. Moreover, both transmembrane potential variations and calcium concentration changes are not limited to the cells that experienced pore formation, but are passed on to neighboring cells in waves 
propagating from the initially affected cells ${ }^{105,106,108}$. This indicates that even though the pores quickly reseal, cellular effects persist over longer time and over larger areas. Sonoporation can additionally lead to structural modifications such as shrinking of the cell ${ }^{110}$, formation of blebs ${ }^{111}$, changes in cytoskeletal orientation ${ }^{112}$ and changes in cellular proliferation ${ }^{105,113}$. Therefore, even though cells remain viable after successful resealing, they may still be altered over a longer term, and could progress towards apoptosis later on ${ }^{100,105}$.

In some studies, enhanced delivery was seen to last during several hours ${ }^{114,115}$. However, membrane pores that stay open during such extended periods of time will surely result in cell death, which is why other mechanisms must be at play here. One potential mechanism is the opening of cellular tight junctions in between cells ${ }^{27,116,117}$. These temporal openings make the cell layer more permeable without affecting cellular integrity and have proven to prolong the transport over a confluent cell layer for up to 30 to $60 \mathrm{~min}^{27}$. They are thought to arise from changes in the cytoskeleton as a result of the shear stress induced by oscillating microbubbles and could be an indirect result of microbubble sonoporation ${ }^{27,117}$. Alternatively, protein-based studies have suggested that ultrasound is able to reduce the protein expression of efflux pumps such as P-glycoprotein, which is normally expressed by the cells that make up the blood-brain-barrier and by certain types of tumor cells ${ }^{118,119}$. Downregulation of P-glycoprotein due to ultrasound exposure will ultimately result in a prolonged intercellular presence of drugs as well.

Another mechanism that enhances drug delivery using microbubbles and ultrasound and that could sustain drug influx over longer periods of time, is the increased incidence of endocytosis ${ }^{115,120-122}$ (figure 6B). Endocytosis is a process that occurs naturally and allows cells to take up important macromolecules from their surroundings. Microbubble oscillations have been shown to promote this process as a result of cell membrane deformation that these oscillations can cause ${ }^{24,103,121}$. Enhanced endocytosis has shown to be more involved in the uptake of larger molecules (>155 kDa) that would have trouble entering the cell through sonoporation due to their low diffusivity ${ }^{120}$. However it has also been proposed that enhanced endocytosis could, in part, be due to membrane disruption by sonoporation, and that both phenomena are actually linked ${ }^{103,123}$. Indeed, studies have reported that small pores created in the cell membrane are often sealed through endocytosis ${ }^{24,123,124}$. Moreover, the calcium-influx resulting from sonoporation, induced the formation of endocytic vesicles as well ${ }^{125}$.

Recently, a novel mechanism called sonoprinting was proposed ${ }^{92}$ (figure 6C). Sonoprinting is defined as the direct deposition of nanoparticles along with parts of the bubble shell onto cell membranes upon applying ultrasound to nanoparticle-loaded microbubbles. It was shown that these patches remain associated with the cell membrane over several hours before finally being internalized. This phenomenon was only seen when nanoparticles where physically coupled onto the bubble surface, 
either via linking drug-loaded nanoparticles to the microbubble shell or via direct incorporation into that shell, and not simply co-administered. Various other studies have reported similar phenomena where they saw fragments of the bubble shell and attached nanoparticles remaining on cell surfaces ${ }^{30,85,126}$. In a follow-up study, high-speed imaging was used to elucidate this sonoprinting phenomena further and it was found that the occurrence of these patches on the cell membrane are most likely due to secondary radiation forces between microbubbles that drag nanoparticles along in their wake ${ }^{127}$. This mechanism can be useful to provide a local reservoir of drug-loaded nanoparticles in the vicinity of the target tissue from where the drugs can leak out and can be taken up by surrounding cells.

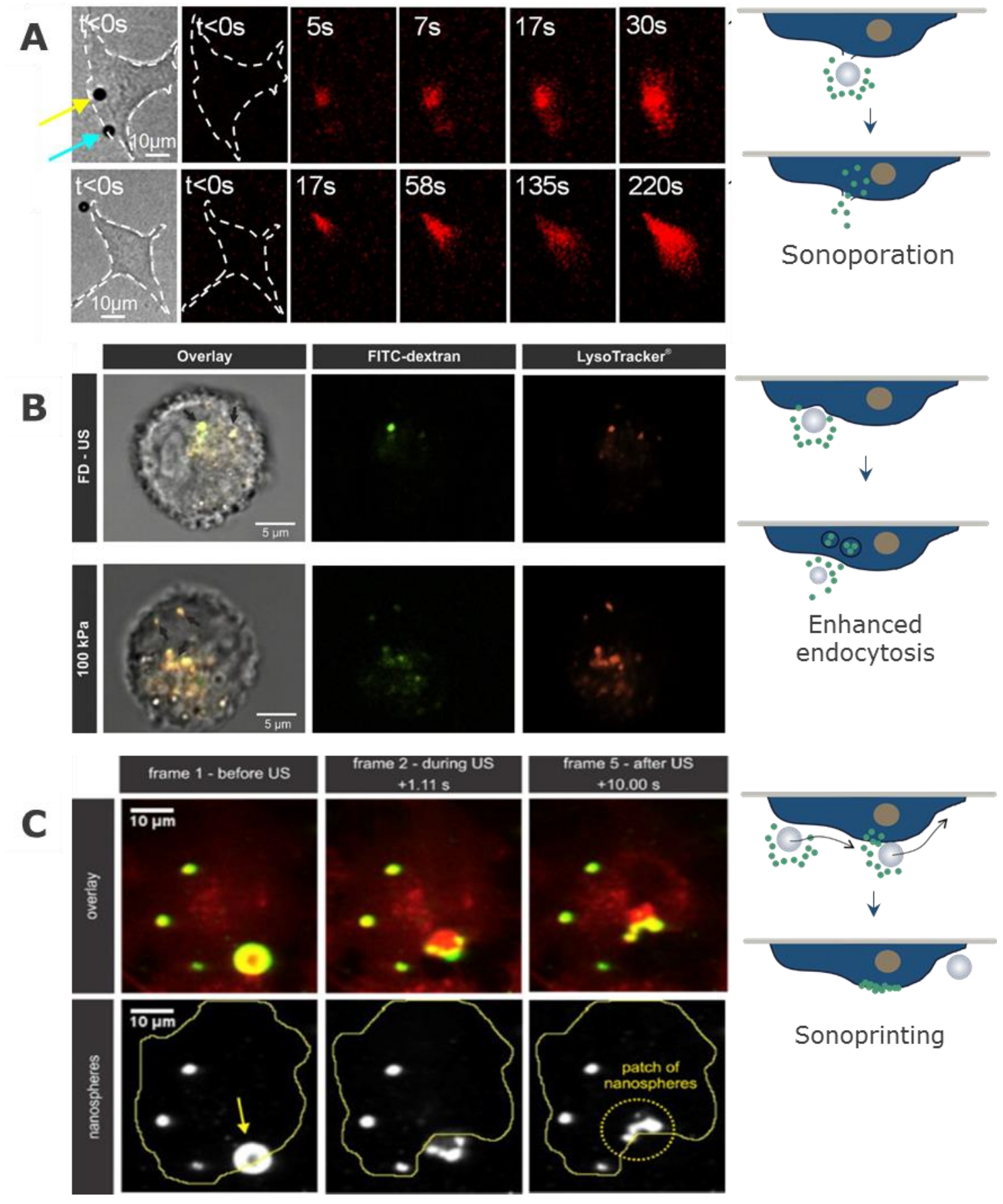


Figure 6. Mechanisms of microbubble-induced drug delivery at the single-cell level. (A) Sonoporation. Upon insonation with a $1.25 \mathrm{MHz}$ frequency, $170 \mathrm{kPa}$ acoustic pressure ultrasound wave for a total ultrasound duration of $8 \mu \mathrm{s}$, the microbubbles create small pores in the cell membrane allowing the otherwise impermeable PI to leak into the cells. In the top image, 2 microbubbles create side-by-side pores, of which the larger of the two microbubbles (indicated with the yellow arrow) leads to more PI influx, presumably due to the creation of a larger pore. (B) Enhanced endocytosis. FITC dextrans are taken up trough endocytosis, evident from the co-localization with lysotracker (i.e.an endo-lysosomal marker), both with and without ultrasound. However applying ultrasound at center frequency of $1 \mathrm{MHz}$, a pulse length of $2000 \mathrm{cycles}$, a driving pressure of $100 \mathrm{kPa}$ and a repetition rate of $125 \mathrm{~Hz}$ for a total duration of $5 \mathrm{~s}$, increases the amount of FITC dextrans inside the lysosomes, indicative of microbubble-induced enhanced endocytosis. (C) Sonoprinting. Sonoprinting can be seen when fluorescently labeled nanospheres are coupled onto microbubbles through chemical linking. After ultrasound radiation of $1 \mathrm{MHz}$ center frequency, an acoustic pressure of $300 \mathrm{kPa}$, a pulse length of 1000 cycles and a repetition rate of $100 \mathrm{~Hz}$ for a total duration of $5 \mathrm{~s}$, a patch of nanospheres is left on the surface of the cell. Adapted from Fan et al. ${ }^{106}$ for (A); De Cock et al. ${ }^{121}$ for (B) and De Cock et al. ${ }^{92}$ for (C) with permission from Elsevier.

The potential of each of the above mentioned mechanisms to enhance the delivery of a therapeutic agent, will rely at first instance on the drug itself. Sonoporation can provide direct access to the cellular cytoplasm and, as such, circumvent several potential biophysical barriers. Nevertheless, since it relies on passive diffusion, this approach will only be suitable for drugs with a relatively small molecular weight, since it is unlikely that larger drugs such as nanoparticles, proteins and genetic drugs will be able to sufficiently diffuse into the cell before the pores have resealed. This was confirmed by studying the contribution of pore formation and enhanced endocytosis on the uptake of molecules of various sizes ${ }^{120,121}$. Indeed, endocytic uptake can theoretically occur for small and large molecules and even for nanoparticles. Larger drug constructs like nanoparticles can also be efficiently delivered via sonoprinting after coupling them to the microbubble surface. Uptake into the cells occurs through internalization of the local patch of nanoparticles. Alternatively, the drugs could leak out from the nanoparticles themselves before endocytosis of the drug-carrier occurs. At this point the drug should have the right physico-chemical characteristics to penetrate into the deeper layers of the tissue and through the cell membranes to exert its function. This approach could be particularly interesting for chemotherapeutic drugs that have sufficient potency but have dose constraints due to off-target effects.

\section{Acoustic settings influencing microbubble-cell interactions and drug uptake mechanisms}

Which of the above mentioned mechanism will be primarily at play will depend on the acoustic settings used, i.e. on the ultrasound frequency, the acoustic pressure, the pulse duration, the pulse repetition frequency, and the duration of insonation. Any one of this large number of ultrasound parameters will largely affect these drug delivery processes by influencing microbubble-cell interactions. Most studies report ultrasound center frequencies around $1 \mathrm{MHz}$, because it roughly matches the resonance frequency of the majority (volume weighted) of bubbles in standard microbubble formulations ( $\pm 3 \mu \mathrm{m}$ in size).

However in terms of acoustic pressures, pressures ranging from $80 \mathrm{kPa}^{71}$ to $1.1 \mathrm{MPa}^{101}$ are used to enhance drug uptake. These pressures span over both stable and inertial cavitation regimes. While the 
frequency and acoustic pressure determines which cavitation regime will occur, the pulse duration controls the time period over which microbubbles can exert an effect. Understanding the interplay between both parameters is essential in tuning the desired drug delivery mechanism. Rather short pulses and high pressures are usually employed for sonoporation purposes ${ }^{27,109,128}$. Upon utilizing targeted microbubbles to ensure a close microbubble-cell contact, Kooiman et al. ${ }^{71}$ demonstrated that sonoporation is induced whenever the relative microbubble vibration amplitude exceeds 0.5 . They showed that this could already be achieved in the stable cavitation regime. The disruption (temporary or permanent) of cell membrane during stable cavitation, is believed to be due to pushing and pulling of the vibrating microbubble on a cell membrane and to the formation of microstreaming that can exert shear stress on the cell membrane ${ }^{5,69}$. Larger shear stress have been shown to correlate with higher propidium iodide uptake, which shows that the pores either stay open longer or are larger. ${ }^{27}$ Likewise, larger holes ${ }^{129}$, higher sonoporation efficiency ${ }^{40}$ and spatiotemporal control over pore formation ${ }^{106}$ can be achieved by using two successive sonoporation pulses with a different pressure. Furthermore, the direct creation of holes in the cell membrane by shockwaves and microjets was observed when higher pressures resulting in microbubble fragmentation were used ${ }^{66,70}$. To obtain a long-term drug delivery increase, enhancement of the endocytic pathway can be induced ${ }^{115,120,121}$. To elucidate which parameters leads to the most prominent endocytic uptake, De Cock et al. ${ }^{121}$ compared the contribution of endocytosis to that of sonoporation on uptake of FITC-dextrans under various acoustic pressures. They showed that when the acoustic pressure is rather low (100-200 kPa) enhanced endocytosis is the dominant mechanism whereas the fraction of sonoporated cells increases with increasing acoustic pressure. This was confirmed by studies using low acoustic pressures to promote endocytosis as well ${ }^{115,130}$.

Whenever the pulse length is increased, radiation forces will come into play. The primary radiation force can push the microbubbles towards target cells and ensure a closer cell-microbubble contact, which stimulates both sonoporation and endocytosis. Secondary radiation forces result in attractive forces between microbubbles, which lead to microbubble aggregation and coalescence ${ }^{129}$. The displacing forces can only move the microbubbles over a substantial distance when the microbubbles are under the influence of ultrasound during a sufficient amount of time, which is why this phenomenon is mostly observed during long pulses (>100 cycles) ${ }^{25}$. Secondary acoustic radiation forces have proven to be essential to achieve sonoprinting ${ }^{127}$. In an attempt to elucidate the impact of acoustic settings on sonoprinting, it was found that increasing the acoustic pressure (>300 $\mathrm{kPa}$ ) and number of cycles (>100 cycles) will result in higher sonoprinting rates. This can be explained by the fact that the released nanoparticles are dragged along with translating microbubbles, resulting in nanoparticle accumulation on the cell membranes. However such translating microbubbles have 
previously been related to cell death in in vitro studies ${ }^{102,129}$. In fact, both an increase in acoustic pressure and in ultrasound pulse length have been positively correlated with cell death ${ }^{98,128,129}$. It is therefore important to maintain a balance between toxicity and drug delivery efficacy and assess the risk-benefit for each treatment goal.

Finally, other ultrasound parameters such as pulse repetition frequency and total exposure duration become increasingly important in the in vivo situation. The pulse repetition frequency will determine the time for microbubble replenishment in between pulses, while the total radiation time will determine which fraction of the microbubbles was able to reach the target site within the duration of the treatment ${ }^{13,131}$. Due to the dependency on blood flow velocity, the pulse repetition frequency as well as the total exposure time should be fine-tuned to the characteristics of the target site, to allow a maximal replenishment in between the ultrasound pulses and an optimal microbubble response in order to maximize therapy.

\section{The impact of microbubbles characteristics on drug delivery}

Often, the effect of microbubbles properties on the drug delivery mechanisms are largely overlooked. Yet, microbubble-related factors could be of equal importance as compared to the acoustic settings in the drug delivery outcome and are therefore also worth investigating. As discussed before, microbubble size is the crucial factor in determining the microbubble's resonance frequency. The therapeutic contribution of microbubbles that do not oscillate at the same amplitude as the resonant bubbles is hard to predict and might affect the balance between inducing therapeutic effects and limiting tissue damage. This once again highlights the importance of more acoustically uniform microbubble formulations for drug delivery applications.

Additionally, microbubble concentration will have a substantial impact, given that the complex interplay between microbubbles can influence the microbubble response. Indeed, higher microbubble concentrations lead to increased microbubble coalescence and aggregation under the influence of secondary radiation forces, while more microbubble translation in the direction of the traveling ultrasound wave due to primary acoustic radiation forces was seen at lower microbubble concentrations $5,81,87$. Furthermore higher microbubble concentrations yield acoustic shielding of microbubbles further away from the transducer, causing a variable microbubble response ${ }^{132}$. Several studies, performed with pressures aiming at inertial cavitation $(600 \mathrm{kPa})$, investigated the impact of the bubble-to-cell ratio on sonoporation and have found that the sonoporation efficiency increased with a higher microbubble to cell ratio with a limit of 4 microbubbles per cell to avoid irreversible sonoporation ${ }^{129,133}$. Consequently, increasing the possibility for bubble-cell interactions augments the chances of efficient drug delivery, however there is a trade-off with cell viability at higher microbubble 
concentrations ${ }^{98}$. Probably even more important than the amount of microbubbles per cell is the microbubble-cell distance, since this will determine the magnitude of the effect a cavitating microbubble can have on a cell. In this respect, studies have tried to elucidate how close the microbubble and the cell need to be and found that this distance should not exceed the microbubble radius $^{94,133,134}$. Nonetheless, shock waves that occur during inertial cavitation can travel over longer distances and affect cells from further away ${ }^{24,135}$.

In in vitro set-ups, a close microbubble-cell contact is often ensured by allowing the microbubbles to float against a cell-carrying substrate $e^{14,71,136}$, which could explain why short ultrasound pulses are sufficient for intracellular uptake in these kinds of studies ${ }^{71,100}$. Even so, in more complex, free-flowing systems, longer pulses might be needed to increase the possibility of close microbubble-cell interactions. To ensure such close microbubble-cell proximity in vitro as well as in vivo, molecularly targeted microbubbles are most often utilized ${ }^{71,102,137}$. These microbubbles contain specific ligands on their shell, which bind to receptor molecules on target cells ${ }^{126,138}$. Note that, as discussed above, modifying the microbubble shell in this way may influence the microbubble's acoustic properties. It was for instance seen that upon applying long pulses on targeted microbubbles that are bound to an underlying surface, secondary Bjerkness forces cause the microbubbles to deform to a prolate shape in the direction of neighboring bubbles at low pressures, before releasing from their target at higher pressures ${ }^{139}$. Other studies have confirmed that a smaller displacement occurred when targeted microbubbles are used ${ }^{102}$. Therefore, drug delivery and associated cell death might be significantly different when targeted microbubbles are used instead of non-targeted microbubbles under the same ultrasound conditions ${ }^{102}$.

\section{Part 3: Translation to preclinical and clinical studies}

Several in vivo studies have been utilizing microbubbles and ultrasound for drug delivery with promising results. However, the lack of optical transparency prevents the use of microscopy-based techniques that are commonly used to study microbubble behavior and microbubble-cell interactions. It is therefore not straightforward to determine the behavior of the microbubbles and how to maximize drug release in vivo, which is reflected in the plethora of different ultrasound parameters being used in these kinds of studies. Yet, a better understanding of the microbubble dynamics in the complex in vivo environment is of vital importance to maximize the therapeutic effects while minimizing adverse effects.

\section{The gap between the in vitro and in vivo situation}

Although a single-microbubble single-cell approach results in a better understanding of the biophysical mechanisms involved, more complex models are crucial to better represent the in vivo environment. 
It is therefore important to comprehend the inherent differences between in vitro and in vivo that will affect microbubble behavior.

\section{Vascular confinement}

A major drug delivery barrier in vivo is the endothelial vessel wall since microbubbles are too large to extravasate through the endothelial fenestrae. Several studies have investigated the effects of vascular confinement on oscillating microbubbles in vitro and in vivo. Due to the damping effect of nearby vessel walls, the maximum oscillation amplitude of microbubbles is less than in free space under the same ultrasound driving conditions ${ }^{140-143}$. Since the maximum amplitude of oscillation has a direct impact on drug release as well as on the various microbubble-cell interactions, it will affect the entire mechanism underlying the drug delivery process. Moreover, the viscosity of blood is approximately 3 to 4 times higher than the viscosity of water-based buffers, as a result the pressure threshold for inertial cavitation was reported to be higher in blood ${ }^{144}$.

Additionally, blood flow velocity and viscosity varies greatly within different sized vessels, and even more in the strongly altered microcirculation of a tumor ${ }^{145}$. Even within a vessel, flow velocity generally decreases from the center to the edges of the vessel and braches within vessels will cause intricate flow patterns ${ }^{146}$, leading to regions of high and low probability of microbubble contact with the vessel wall and local microbubble accumulations. Consequently, the time a microbubble is exposed to ultrasound and therefore its potential to induce local bio-effects, may be different for each microbubble. Similarly, the microbubble concentration will fluctuate within the target area. Therefore, drug delivery mechanisms that rely on the interaction between adjacent microbubbles might be affected as well. It remains to be seen if targeting strategies can ensure the close contact needed for these kinds of interactions to occur. Moreover since nearby membranes create additional microbubble translation due to secondary radiation forces, the microbubbles behavior may vary when a microbubble is oscillating within a large vessel as compared to when it is closed in by nearby capillary walls.

Ultrasound-mediated microbubble cavitation is furthermore able to locally alter tissue perfusion. Vasoconstriction and even a complete shut-down of microvascular blood flow has been reported when violent microbubble collapses are induced by relatively high ultrasound pressures of $1.6 \mathrm{MPa}^{147}$. This temporary vascular shut-down can hamper drug influx and even lead to ischemic damage ${ }^{148}$. This has led to a growth delay in tumors, caused by the endothelial cell apoptosis and local inflammation resulting in platelet activation and occlusion of the microvasculature ${ }^{149-151}$. In contrast, an increased perfusion after microbubble-assisted ultrasound treatment was also described in literature ${ }^{152-154}$. Rix et al. ${ }^{153}$ reported augmented signal enhancement in tumors after repeated microbubble injections and mentioned cavitation-assisted mechanical opening of closed microvessels as one of the possible 
reasons. Belcik et al. ${ }^{152}$ stated that microbubble cavitation could reverse tissue ischemia in the hind limb muscle of mice. Yet, it could not be completely ruled out that thermal ultrasound effects caused this enhanced perfusion. Likewise, since perfusion imaging was only performed 10 to 15 min after therapy, it is possible that the vasodilation resulted from a vascular rebound effect after an initial vasoconstriction $^{148}$.

\section{Tissue structure}

Before reaching its in vivo target site, ultrasound will be subject to attenuation and reflection due to tissue viscoelasticity and inhomogeneity. Therefore, microbubbles within this target site will not receive the same ultrasound energy as they would in an in vitro setting. Furthermore, this attenuation is highly dependent on individual tissue structures and can hence vary between patients.

Cells grown in a three-dimensional system instead of a two-dimensional monolayer will have altered phenotypes ${ }^{155-157}$ that can lead to variations in their response to ultrasound and microbubbles. Even on an in vitro level, it was already shown that endothelial cells grown under flow condition are less responsive to the same ultrasound regime than cells grown under static conditions ${ }^{109}$. The authors attribute this finding to alteration of the cell's phenotype resulting in extended F-actin stress fibers, increase in cytoplasm viscosity and cell membrane rigidity that make the cell more resistant to external shear forces.

Another major difference is the lack of rigid membranes in an in vivo setting which has been shown to alter the microbubbles acoustic response significantly. For example, microjets have been reported to occur due to a bubble collapse in the direction of the cell-carrying stiff wall, which were able to puncture the cells $s^{66,70,158}$. However, since soft tissue is much less elastic, some of the non-spherical effects seen in vitro may not be present to the same extent, or not at all in vivo ${ }^{5}$. Likewise, shedding of shell material was seen to be directed away from the supporting membrane ${ }^{36}$, which might not be the case in the clinical situation.

\section{In vivo reports of drug delivery enhancement using microbubbles and ultrasound}

Since the in vivo situation is often regarded as a black box system, especially in comparison to the highly controlled environment of an in vitro study, most studies only report on the outcome of the treatment. Even so, promising therapeutic results have already lead to a first clinical trial using gemcitabine, i.e. a small molecule chemotherapeutic, in combination with microbubbles and ultrasound in 10 patients with inoperable pancreatic tumors ${ }^{159}$. The results of this study indicated that the treatment was well tolerated, and that the tumor diameter decreased more compared to gemcitabine treatment alone (based on results from historical patients). Other clinical trials reported on the opening of the blood-brain barrier to allow chemotherapeutic treatment of brain tumors, and 
their preliminary findings have indicated that the procedure is safe and well-tolerated in patients ${ }^{160-}$ ${ }^{162}$. Notwithstanding these encouraging results, they are only primary results on a selected group of patients and there are a lot of variables that remain undiscussed. Moreover, some results do not correlate well with what was previously established. For instance, the clinical study by Carpentier et al. ${ }^{160}$ reports safe opening at pressures that have been reported to cause extensive adverse effects in small laboratory animals and non-human primates ${ }^{161,163-165}$. They attributed this discrepancy to the size of the human skull which could limit the occurrence of standing waves, the nature of human microvessels or an anesthesia-related difference in physiological conditions ${ }^{160}$. To obtain a conclusive understanding of the significance of these effects, additional research is necessary.

Nonetheless, (pre-)clinical models provide a unique opportunity to study cellular interactions that are difficult to replicate in vitro. These models cannot only confirm the mechanisms that are previously observed, but can also reveal new mechanisms of action. Yet, different evaluation methods are often required, which complicates the interpretation of results. To increase the knowledge on microbubbleassisted drug delivery in vivo, methods to estimate the tissue distribution of the drug in small animals have been described, either on the entire animal using near-infrared fluorescence imaging ${ }^{15,166}$, or by harvesting the organs and extracting the drug separately ${ }^{167}$. MRI has been widely used to monitor blood-brain barrier opening, with the aid of MR contrast agents ${ }^{97,165,168-170}$. This has proven that even the tight-junctions that make up the blood-brain barrier can be opened by microbubble-assisted ultrasound treatment, giving this approach a unique opportunity to channel drugs into the cerebral fluids across this otherwise impermeable barrier ${ }^{68,161,171-173}$. A more detailed analysis of the physiological effects and drug uptake in different organs can be obtained via histology and immunohistochemistry. Using this approach, positive results have been obtained for numerous of microbubble-drug formulations and ultrasound in comparison to the delivery of drugs or nanoparticles alone $\mathrm{e}^{10,15,167,174-176}$. Nevertheless, a number of adverse effects have also been reported, including hemorrhage $\mathrm{e}^{15,171,175}$, local burns ${ }^{16}$, necrosis due to vascular shut-down, formation of thrombi ${ }^{148,177}$ as well as drug accumulation in lungs, liver and kidney. Furthermore, immunohistochemistry has confirmed the down-regulation of P-glycoprotein in endothelial cells and astrocytes that make up the blood-brain barrier due to microbubble-assisted ultrasound treatment ${ }^{170,178}$.

Other studies have tried to retrieve the (model) drug or drug carrier inside the ultrasound-treated tissue using cryosectioning $15,171,175,179$, fluorescence molecular tomography (FMT) ${ }^{116,180}$ or two photon microscopy ${ }^{174}$. These techniques were used to investigate the drug distribution in the tissue and the ability of drugs and nanoparticles to reach more distant parts, beyond the blood vessel. For nanoparticles of approximately $100 \mathrm{~nm}$ in size, average penetration distances of $15 \mu \mathrm{m}^{175}$ to maximally $45-50 \mu \mathrm{m}^{174,175}$ have been reported. Rather large $(177 \mathrm{~nm})$ nanoparticles have also been found to 
penetrate the vessel wall in the brain, but remained just outside the vasculature in this case ${ }^{171}$. Furthermore, Burke et al ${ }^{116,180}$ showed that the nanoparticles can be retrieved inside endothelial cells, indicative of sonoporation, as well as in the extracellular space, indicative of enhanced permeability of the endothelial lining. Different studies have also compared the delivery efficiency of nanoparticleloaded microbubbles to the co-administration approach, and concluded that a higher nanoparticle delivery could be achieved when the nanoparticles are directly coupled onto microbubbles ${ }^{116,180}$. Furthermore, coupled nanoparticles were found to concentrate in discrete regions, as opposed to a more even distribution in case of co-administered nanoparticles ${ }^{180}$. This indicates that the nanoparticles remained grouped in clusters possibly delivered via a sonoprinting-like phenomenon. Another study by Kilroy et al. ${ }^{181}$ used an intravascular ultrasound probe to promote acoustic radiation forces and found fluorescently labeled microbubble shell fragments deposited on the vessel wall, even without the subsequent administration of a 'delivery pulse', i. e. a shorter ultrasound pulse at higher pressure to induce sonoporation. This again indicates that other phenomena than pore formation through sonoporation might be at play in vivo as well.

Finally, the intricate intra- and intercellular signaling pathways that often lack in simple in vitro models, can lead to the uncovering of additional therapy-related effects. For example, the potential induction of sterile inflammation in the brain after blood-brain barrier opening was recently described ${ }^{97}$. Here, it was reported that although no microscopic tissue damage was observed, an increased presence of pro-inflammatory factors was present at the opening site. This acute inflammation was compatible with ischemia or mild traumatic brain injury and could potentially pose a safety treat. Conversely, it could also been linked to positive effects, such as the augmented removal of amyloid plaques, which play an important role in certain neurodegenerative diseases. Another drug delivery approach that was primarily investigated in vivo, is the injection of microbubble-microdroplet clusters that undergo a phase shift upon ultrasound radiation and create large bubbles, which can temporarily block capillaries $^{182}$. This system was used in combination with numerous commercially available drug formulation and has shown enhanced therapeutic effects in small animal studies ${ }^{183-185}$. Yet, the exact mechanism behind this approach, as well as the safety in clinical translation has not yet been fully clarified. Likely, a variety of other factors will be involved in the complex in vivo situation, which is why it was very recently suggested to refer to ultrasound and microbubble-mediated enhanced drug delivery in general, as 'sonopermeation' ${ }^{186}$.

\section{Acoustic parameters used in in vivo studies}

Similar to in vitro studies, a high variability in acoustic parameters can be found in in vivo studies. As compared to in vitro reports, in vivo studies are generally performed at higher acoustic pressures (hundreds of $\mathrm{kPa}$ to even $7 \mathrm{MPa}^{15,116,175}$, as measured in pure water) and longer pulse durations 
(hundreds to ten thousands of cycles ${ }^{15,187}$ ), rationalized by the higher level of attenuation and the lack of immediate microbubble-cell contact. Also, the overall exposure time is usually much longer (up to $10 \mathrm{~min}^{174}$ ) to allow a maximum amount of microbubbles to be affected during treatment. Authors have suggested that inertial cavitation is needed to provide adequate intratumoral drug delivery in vivo $^{175,188}$, but this has not yet been thoroughly examined. In contrast, stable cavitation is said to be sufficient for blood-brain barrier opening without tissue damage ${ }^{163,165,189,190}$. Furthermore, while the use of longer cycles may be crucial in vivo to elicit acoustic radiation forces and sonoprinting effects needed for delivery of larger drugs, short pulses (5 cycles) emitted at high pulse repetition frequencies (1.25 MHz) have shown to result in a more uniform distribution of small molecules taken up through thigh junctions ${ }^{187}$. Recently, follow-up studies on the report of Kovacs et al. ${ }^{97}$, which claimed that microbubble-assisted opening of the blood-brain barrier could lead to a sterile inflammatory response, have pointed out that the outcome greatly depends on both the microbubble- as the ultrasound parameters, and that strict control over these factors is crucial ${ }^{191-193}$. This highlights once again the importance of control over these parameters, and shows that variations herein may result in completely different interpretations of occurring phenomena. Again, the optimal parameters for each application will depend on the drug itself, on the microbubble response and on the desired therapeutic application.

Most studies have used a trial-and-error approach to optimize the acoustic parameters needed for drug delivery ${ }^{16,194}$, which didn't always match the set of parameters found to be ideal in accompanying in vitro pre-screening studies ${ }^{10,194}$. This strategy is, a priori, ill-suited when dealing with such a large number of parameters. Additionally, many preclinical and clinical ultrasound devices do not allow modification of a number of important ultrasound parameters. The frequency can be changed within the limited bandwidth of the ultrasonic probe and the maximum intensity output can be adjusted, but pulse length is often fixed in clinical machines. Therefore, many in vivo studies report ultrasound output only in terms of mechanical index (MI) or ultrasound intensity in $\mathrm{W} / \mathrm{cm}^{2}{ }^{166}$, which does not reflect all characteristics of an ultrasonic wave. In fact, $\mathrm{MI}$ is only determined by the frequency $(\mathrm{f})$ and pressure (PNP) of the ultrasound wave $(\mathrm{MI}=\mathrm{PNP} / \sqrt{f})$, while pulse length, pulse repetition frequency and total duration are disregarded despite the large impact they can have on the overall effect. Moreover, equal MI values do not necessarily result in equal microbubble behavior, as explained in section 2. Some studies report pulse length (PL) and pulse repetition frequency (PRF) in terms of duty cycle $(D C=P L * P R F)$, but the same value for duty cycle can be obtained by a combination of these terms which will not affect the microbubble response in the same way as explained earlier on. Therefore, a thorough characterization of the ultrasound system is mandatory, as well as a detailed report of all acoustic settings (frequency, pressure, pulse length, pulse repetition frequency and total 
duration of the treatment). This might allow a more straightforward comparison between research results from different groups and it might make the in vitro to in vivo translation more straightforward.

As mentioned above, most clinical and preclinical ultrasound imaging systems have a few fixed settings, mostly in terms of pulse length, which are not ideal for drug delivery purposes. In a study with the Vevo 2100 , the power doppler mode was employed to improve vascular permeability ${ }^{174}$. However this only allows very short pulses, at a frequency far from the resonance frequency in this case. Another study by Luo et al. ${ }^{10}$ used a flash mode on the Philips iU22 with similar issues. Nowadays, programmable ultrasound research systems, can be used to generate the longer pulses needed for drug delivery ${ }^{195}$. Clinical transducers often have a frequency range starting at $2 \mathrm{MHz}$, while for most microbubble formulations, $1 \mathrm{MHz}$ ultrasound matches the overall resonance frequency the best. For preclinical small-animal scanners, this difference is even more profound, as these type of transducers typically only start at frequencies of $10 \mathrm{MHz}$ to allow sufficient resolution on ultrasound scans of small laboratory animals. It would be possible to formulate monodisperse microbubbles that have an ideal size for the higher frequencies of commercial clinical scanners, yet, smaller microbubbles allow less loading of drug molecules and will be intrinsically less efficient due to reduced backscatter and higher damping.

\section{A unified approach to evaluate microbubble behavior and associated drug uptake in vitro and in vivo}

To monitor microbubble and ultrasound-assisted treatment in vivo, researchers have used conventional B-mode imaging to study the presence and destruction of microbubbles, thermocouples to evaluate temperature changes, MRI with MRI contrast agents to check biodistribution and CT and PET techniques to examine the biological effect ${ }^{13,131}$. However these techniques monitor either physical parameters or secondary biological effects while techniques to study direct biophysical aspects in vivo are lacking. Moreover, these techniques often have a rather poor resolution and cannot be used in real-time ${ }^{131}$.

A more recent approach is to use passive cavitation detection (PCD) to monitor microbubble cavitation behavior in real-time and this can be extended to form cavitation maps in $2 D$ and $3 D^{13,131,188,196,197}$. In $\mathrm{PCD}$, a single-element transducer is used to detect scattered signals from the microbubbles which provides information on the microbubble dynamics in the treated region. Therapy monitoring using PCD is based on the hypothesis that the cavitation behavior of microbubbles in the target area is directly related to the overall therapeutic outcome ${ }^{198}$. In this way, therapeutic bio-effects could be linked to a certain cavitation dose, and the system can be used to monitor whether, when and where this dose is achieved. Since the microbubbles oscillate non-linearly, their backscattered signal will not only contain the incident frequency but also harmonics of the insonifying frequency. A frequency 
spectrum can be derived from the acoustic signals received by the PCD transducer via a fast Fourier transform and can be used to determine the acoustic behavior of the microbubbles in the focus region. If the spectrum predominantly consists of harmonics and subharmonics, microbubbles are mostly in the stable oscillation regime. Contrarily, if broadband noise prevails, microbubbles are cavitating inertially. Since single-element transducers can only provide limited information on the location and amount of cavitation events, new techniques making use of array transducers have recently been introduced ${ }^{131,199}$. Array transducers allow to analyze the acoustic emissions from microbubbles in individual voxels within the ultrasound treatment zone independently, and can be used to form a cavitation map of the region of interest. This technique is called passive acoustic mapping (PAM) and is especially useful to determine where the regions of highest cavitational energy are ${ }^{13,131}$. One example where PAM can be beneficial is to assess patient-specific attenuations in ultrasound radiation of the brain, to ensure that the desired microbubble behavior is present within the treatment zone ${ }^{198}$.

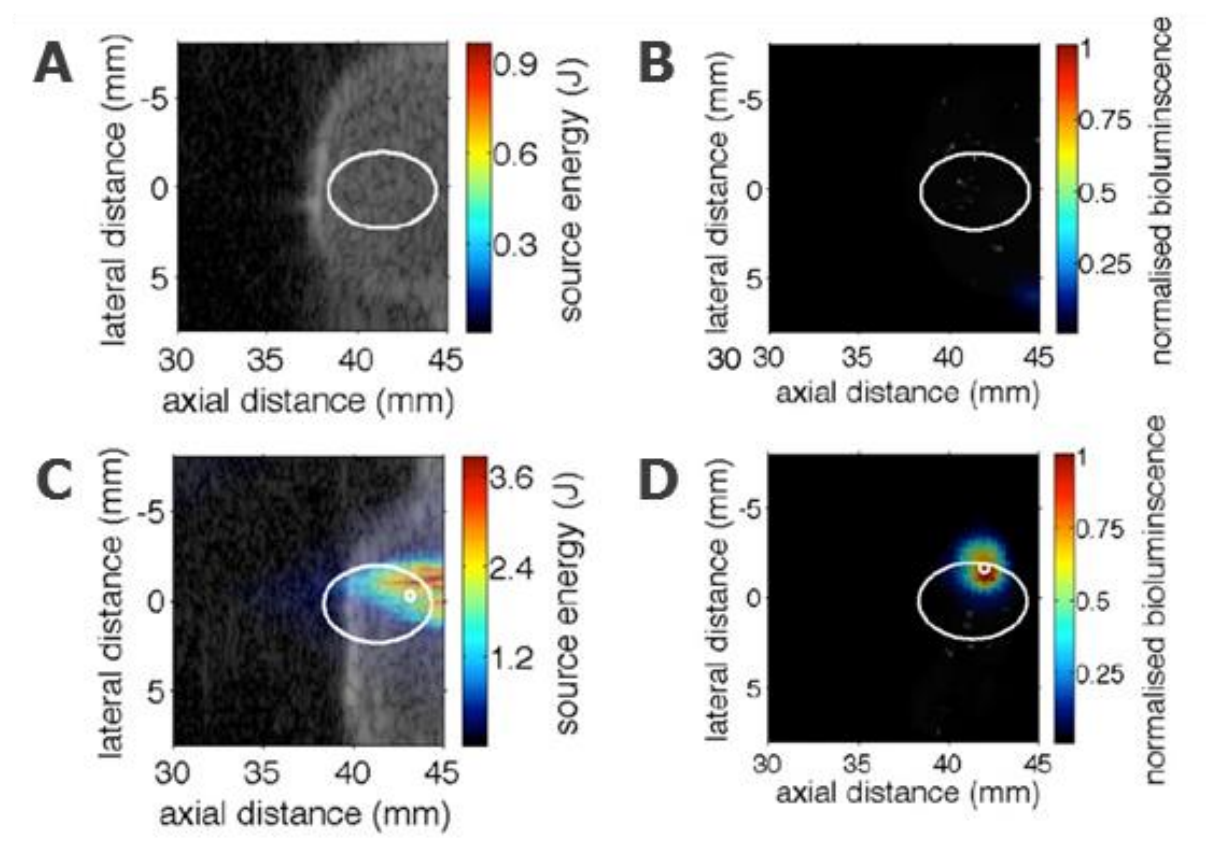

Figure 7: Murine tumors exposed to 80 pulses of $0.5 \mathrm{MHz}$ ultrasound with a $100 \mathrm{~ms}$ pulse length, $1.2 \mathrm{MPa}$ peak negative pressure and a $3 \mathrm{~s}$ pulse repetition period in the presence of systemically administered luciferin-containing liposomes, with (C\&D) or without (A\&B) Sonovue ${ }^{\circledR}$ microbubbles. A\&C: Overlay between B-mode images and passive acoustic maps of the tumor volume showing the regions of cavitation. B\&D: Bioluminescence measurements of the same location, indicating luciferin release from the liposomes. From $C$ and $D$ it is clear that inertial cavitation of the microbubbles, as evident from the source energy of broadband noise, corresponded to luciferin release from the cavitation-sensitive liposomes. Adapted from Choi et al. ${ }^{131}$ with permission from IOP Publishing. CInstitute of Physics and Engineering in Medicine. All rights reserved.

Several studies have already used passive cavitation detection or passive acoustic mapping to link the cavitation behavior of microbubbles to therapeutic bio-effects, both in vitro ${ }^{13,175}$ as in vivo ${ }^{131,188,200}$. One study in particular showed that therapeutic activity of oncolytic viruses can only be detected when the microbubble emission spectra displayed signs of inertial cavitation ${ }^{200}$. Inertial cavitation was also required to stimulate liposomes break-up of specific drug-releasing liposomes co-administered with 
microbubbles ${ }^{131,188}$ (figure 7). It was also shown that therapeutic activity was mostly present at sites where the highest acoustic activity was measured, although this was not always at the focus of the transducer ${ }^{131}$. This was assumed to result from a non-uniform distribution of the microbubbles in the tumor owing to its irregular microvasculature, which is represented in the maps as well. Studying cavitation activity over time allowed the authors to monitor the impact of different ultrasound parameters in vivo. This revealed that a sufficiently long pulse repetition period (i.e. in the order of seconds) was advantageous as it ensured microbubble replenishment at the target area ${ }^{131}$. It was also shown that the cavitation life-time of the microbubbles is strongly shortened when higher pressures are applied which makes the use of longer pulse lengths redundant ${ }^{131,188,198,201}$. Recently, a real-time feedback system was developed to control the opening of the blood-brain barrier in-situ ${ }^{189,190}$. With this loop control, acoustic emissions from microbubbles at the target site could directly be used to tune the ultrasound parameters to precisely control the magnitude of blood-brain barrier opening and the resulting drug delivery.

The implementation of passive acoustic mapping is nonetheless not straightforward. Only a limited number of clinical systems allow to implement this technique ${ }^{196}$. A high computational power and specially designed algorithms are required to process the data. As mentioned before, one would ideally use a second transducer to capture the echoes from the microbubbles at a higher frequency to study the harmonics and broadband content without interference from scatter at the fundamental frequency. Occasionally, a system of even 3 transducers is used, where one provides imaging, the second sends out the therapeutic ultrasound radiations and the third records the passive cavitation signals ${ }^{131,175}$. Synchronized passive recording using a single transducer should be possible but only if the pulses are sufficiently short ${ }^{196}$. Only very short pulses guarantee that the same microbubble population is still present, which could form a problem for the typically longer pulses needed in drug delivery. Another difficulty of longer pulses is the relatively poor axial resolution which makes it difficult to avoid interference from regions of high bubble density with regions of low bubble density along this axis. For example, imaging cavitation in the myocardium proved nearly impossible due to signal smearing from the highly perfused nearby ventricle ${ }^{198}$. Even though there are still some issues to be resolved here, passive cavitation detection would allow researchers to directly link microbubble dynamics to therapeutic effects in real-time and could help bridge the gap between studies focusing only on acoustic behavior of microbubbles on the one hand and studies focusing only at therapeutic outcome on the other hand.

\section{Concluding remarks}

Microbubble and ultrasound-driven drug delivery has been extensively studied with promising outcome which even resulted in the first clinical trials. A critical issue remains the prediction and 
control of therapy in different models. Having a basic understanding of the physics of microbubble dynamics is necessary to understand why acoustic settings and microbubble characteristics are crucial and how they can affect microbubble behavior. However, from literature, it is obvious that a plethora of parameters are used, leading to an equal variety in bio-effects. Similarly, microbubble polydispersity can be the cause of inconsistent results since different sized microbubbles will respond differently to the same ultrasound driving pulse. The narrow bandwidth resulting from the long pulses needed for drug delivery makes this issue even more essential. Moreover, inherent features of in vitro set-ups may alter the microbubble behavior in ways that are not representative for the clinical situation. Hence, translating microbubble dynamics and therapeutic outcome from in-depth in vitro studies to in vivo results from has proven to be very challenging.

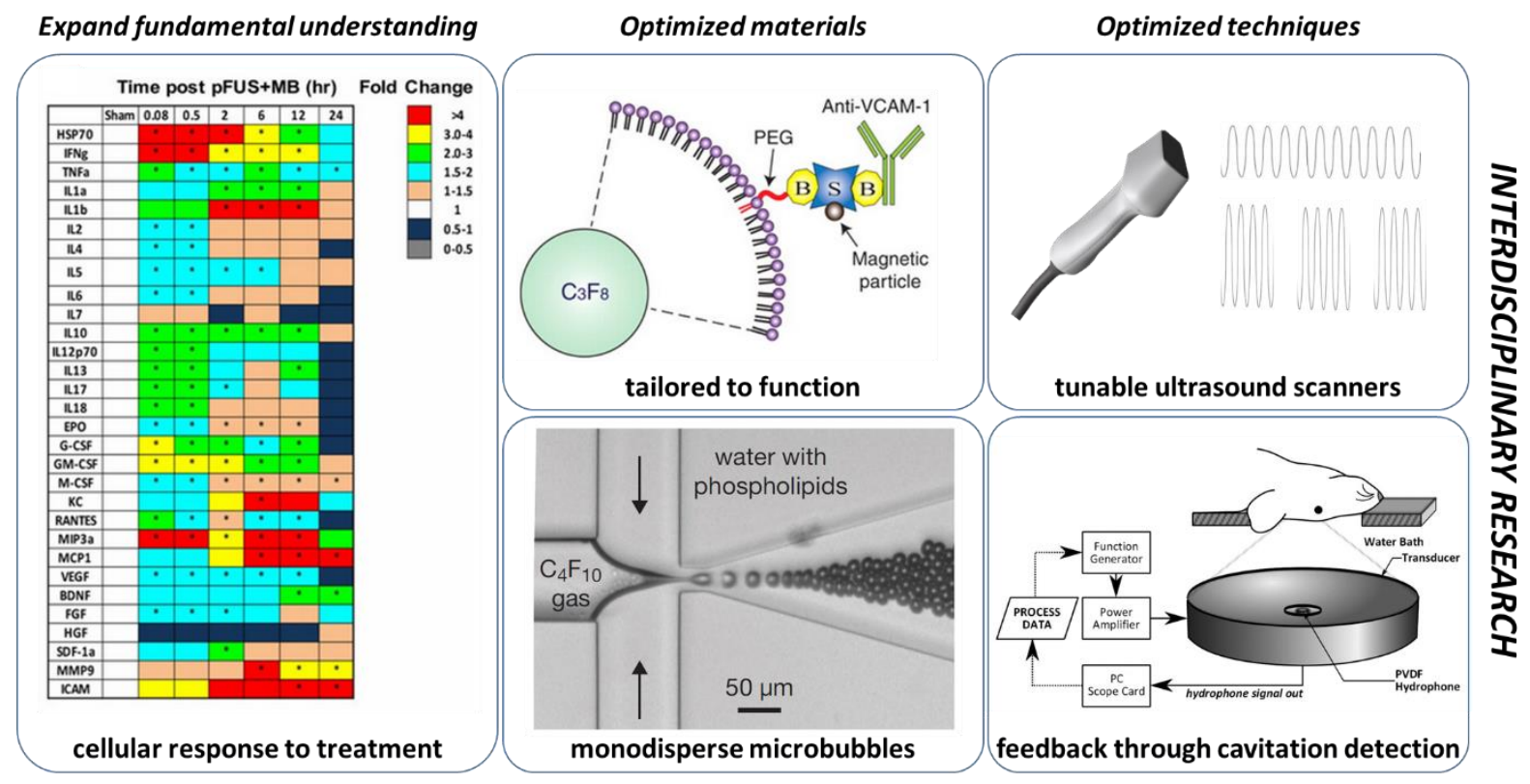

Figure 8: Future directions. Left panel: Expanding the fundamental understanding of microbubble behavior and the cellular response to treatment in vitro as well as in vivo, will remain crucial for future development. For example, protein analysis of several pro-inflammatory factors in rat brain following treatment with microbubbles and ultrasound by Kovacs et al., revealed that this treatment can lead to a sterile inflammatory response compatible with ischemia or mild traumatic brain injury, raising possible safety questions for clinical translation. Middle panel: For therapeutic purposes, commercial microbubble preparations will be far outperformed by custom microbubbles specifically tailored for targeting, drug loading, multimodal imaging, etc. (top panel) and monodisperse microbubble preparations that result in more robust and predictable microbubble behavior (bottom panel). Right panel: Progress in ultrasound equipment and in-situ monitoring of treatment may lead the way to a more effective and controlled therapy. Figures adapted from Kovacs et al. ${ }^{97}$ (left); Wu et al. ${ }^{18}$ with permission from RSNA (middle panel - top); from Segers et al. ${ }^{202}$ with permission from The Royal Society of Chemistry (middle panel - bottom) and from O'Reilly et al. ${ }^{190}$ with permission from RSNA (right panel - bottom).

Figure 8 shows recent developments and future directions that can help overcome these issues and may eventually pave the way to a more efficient and tailored microbubble-assisted ultrasound therapy. Expanding the fundamental understanding of microbubble behavior and the cellular response to treatment in vitro as well as in vivo, will remain crucial in obtaining new perspectives on previously established concepts. For example, exploring functional effects of microbubble treatment such as 
changes in protein expression, can provide additional insights on the interaction of cavitating microbubbles with cells and tissues. Furthermore, newer models and techniques like monodisperse microbubble formulations, tunable ultrasound scanners and cavitation detection techniques have provided us with different tools to obtain more uniform microbubble responses and to monitor treatment. This could eventually lead to more controllable and predictable microbubble responses and thereby, to more efficient and measurable therapy in the future. Despite these promising developments, implementing these new techniques requires specific knowledge of various fields of expertise, highlighting the need for interdisciplinary partnerships between biologists, chemists, engineers, physicists, programmers, etc. Future work should therefore focus on merging the knowledge from these subdomains to develop an ideal therapeutic agent, together with a clinical ultrasound system that allows simultaneous imaging, treatment and monitoring within one single device useable by all research groups.

\section{Acknowledgements}

Silke Roovers is a doctoral fellow of the Special Research Fund in Flanders, Belgium (BOF-Vlaanderen). The research was also funded through NanoCOMIT, an SBO project granted by the Institute for the Promotion of Innovation through Science and Technology in Flanders, Belgium (IWT-Vlaanderen). The support of both these institutions is gratefully acknowledged. 


\section{References}

1. Studwell, A. J. \& Kotton, D. N. A shift from cell cultures to creatures: In vivo imaging of small animals in experimental regenerative medicine. Molecular Therapy 19, 1933-1941 (2011).

2. Lentacker, I., De Smedt, S. C. \& Sanders, N. N. Drug loaded microbubble design for ultrasound triggered delivery. Soft Matter 5, 2161-2170 (2009).

3. Wells. Ultrasound imaging. Phys. Med. Biol. 51, R83--R98 (2006).

4. Lajoinie, G. et al. In vitro methods to study bubble-cell interactions: Fundamentals and therapeutic applications. Biomicrofluidics 10, (2016).

5. Kooiman, K., Vos, H. J., Versluis, M. \& De Jong, N. Acoustic behavior of microbubbles and implications for drug delivery. Advanced Drug Delivery Reviews (2014). doi:10.1016/j.addr.2014.03.003

6. Segers, T., de Jong, N., Lohse, D. \& Versluis, M. Chapter 5. Microbubbles for Medical Applications. in 81-101 (The Royal Society of Chemistry, 2015). doi:10.1039/9781849737593-00081

7. Sirsi, S. R. \& Borden, M. A. Microbubble compositions, properties and biomedical applications. Bubble Sci. Eng. Technol. 1, 3-17 (2009).

8. Marmottant, P. et al. A model for large amplitude oscillations of coated bubbles accounting for buckling and rupture. J. Acoust. Soc. Am. 118, 3499-3505 (2005).

9. Faez, T. et al. 20 years of ultrasound contrast agent modeling. IEEE Trans. Ultrason. Ferroelectr. Freq. Control 60, 720 (2013).

10. Luo, W. et al. Dual-targeted and $\mathrm{pH}$-sensitive doxorubicin prodrug-microbubble complex with ultrasound for tumor treatment. Theranostics 7, 452-465 (2017).

11. Geers, B. et al. Targeted liposome-loaded microbubbles for cell-specific ultrasound-triggered drug delivery. Small 9, 4027-4035 (2013).

12. Bae, Y. J., Yoon, Y. II, Yoon, T. J. \& Lee, H. J. Ultrasound-guided delivery of siRNA and a chemotherapeutic drug by using microbubble complexes: In vitro and in vivo evaluations in a prostate cancer model. Korean J. Radiol. 17, 497-508 (2016).

13. Crake, C. et al. Passive acoustic mapping of magnetic microbubbles for cavitation enhancement and localization. Phys. Med. Biol. 60, 785-806 (2015).

14. Lentacker, I., Geers, B., Demeester, J., De Smedt, S. C. \& Sanders, N. N. Design and evaluation of doxorubicincontaining microbubbles for ultrasound-triggered doxorubicin delivery: Cytotoxicity and mechanisms involved. Mol. Ther. 18, 101-108 (2010).

15. Snipstad, S. et al. Ultrasound Improves the Delivery and Therapeutic Effect of Nanoparticle-Stabilized Microbubbles in Breast Cancer Xenografts. Ultrasound Med. Biol. 43, 2651-2669 (2017).

16. Cool, S. K. et al. Coupling of drug containing liposomes to microbubbles improves ultrasound triggered drug delivery in mice. J. Control. Release 172, 885-893 (2013). 
17. Cai, X., Yang, F. \& Gu, N. Applications of magnetic microbubbles for Theranostics. Theranostics 2, 103-112 (2012).

18. Wu, J. et al. Efficacy of Contrast-enhanced US and Magnetic Microbubbles Targeted to Vascular Cell Adhesion Molecule-1 for Molecular Imaging of Atherosclerosis. Radiology 260, 463-471 (2011).

19. Stride, E. Physical principles of microbubbles for ultrasound imaging and therapy. Cerebrovasc. Dis. 27 Suppl 2, 113 (2009).

20. Postema, M. \& Gilja, O. H. Contrast-enhanced and targeted ultrasound. World J. Gastroenterol. 17, 28-41 (2011).

21. Cosgrove, D. Ultrasound contrast agents: An overview. Eur. J. Radiol. 60, 324-330 (2006).

22. Stride, E. P. \& Coussios, C. C. Cavitation and contrast: The use of bubbles in ultrasound imaging and therapy. Proc. Inst. Mech. Eng. Part H J. Eng. Med. 224, 171-191 (2010).

23. Goldberg, B. B., Liu, J. B. \& Forsberg, F. Ultrasound contrast agents: a review. Ultrasound Med. Biol. 20, 319-333 (1994).

24. Lentacker, I., De Cock, I., Deckers, R., De Smedt, S. C. \& Moonen, C. T. W. Understanding ultrasound induced sonoporation: Definitions and underlying mechanisms. Advanced Drug Delivery Reviews 72, 49-64 (2014).

25. Ferrara, K., Pollard, R. \& Borden, M. Ultrasound Microbubble Contrast Agents: Fundamentals and Application to Gene and Drug Delivery. Annu. Rev. Biomed. Eng. 9, 415-447 (2007).

26. Hu, Y., Wan, J. M. F. \& Yu, A. C. H. Membrane Perforation and Recovery Dynamics in Microbubble-Mediated Sonoporation. Ultrasound Med. Biol. 39, 2393-2405 (2013).

27. Helfield, B., Chen, X., Watkins, S. C. \& Villanueva, F. S. Biophysical insight into mechanisms of sonoporation. Proc. Natl. Acad. Sci. 113, 9983-9988 (2016).

28. Moonen, C. \& Lentacker, I. Ultrasound assisted drug delivery. Advanced Drug Delivery Reviews 72, 1-2 (2014).

29. Klibanov, A. L., Shevchenko, T. I., Raju, B. I., Seip, R. \& Chin, C. T. Ultrasound-triggered release of materials entrapped in microbubble-liposome constructs: A tool for targeted drug delivery. J. Control. Release 148, 13-17 (2010).

30. Kheirolomoom, A. et al. Acoustically-active microbubbles conjugated to liposomes: Characterization of a proposed drug delivery vehicle. J. Control. Release 118, 275-284 (2007).

31. Li, W., Juan, T., Guo, X. S., Di, X. \& Dong, Z. Microstreaming velocity field and shear stress created by an oscillating encapsulated microbubble near a cell membrane. Chinese Phys. B (2014). doi:10.1088/1674-1056/23/12/124302

32. Doinikov, A. A. \& Dayton, P. A. Maxwell rheological model for lipid-shelled ultrasound microbubble contrast agents. J. Acoust. Soc. Am. 121, 3331 (2007).

33. Tu, J., Guan, J., Qiu, Y. \& Matula, T. J. Estimating the shell parameters of SonoVue ${ }^{\circledR}$ microbubbles using light scattering. J. Acoust. Soc. Am. 126, 2954-2962 (2009).

34. $W u, J$. Theoretical study on shear stress generated by microstreaming surrounding contrast agents attached to living cells. Ultrasound Med. Biol. 28, 125-129 (2002). 
viscoelastic layers. J. Acoust. Soc. Am. 132, 124-137 (2012).

36. Lajoinie, G. et al. Non-spherical oscillations drive the ultrasound-mediated release from targeted microbubbles. Commun. Phys. 1, 22 (2018).

37. Kooiman, K., Vos, H. J., Versluis, M. \& De Jong, N. Acoustic behavior of microbubbles and implications for drug delivery. Advanced Drug Delivery Reviews 72, 28-48 (2014).

38. Humphrey, V. F. Ultrasound and matter-Physical interactions. Progress in Biophysics and Molecular Biology 93, 195-211 (2007).

39. Minnaert, M. On musical air-bubbles and the sound of running water. London, Edinburgh, Dublin Philos. Mag. J. Sci. 16, 235-248 (1933).

40. Song, K. H. et al. High efficiency molecular delivery with sequential low-energy sonoporation bursts. Theranostics 5 , 1419-1427 (2015).

41. Kok, M. P., Segers, T. \& Versluis, M. Bubble sorting in pinched microchannels for ultrasound contrast agent enrichment. Lab Chip 15, 3716-3722 (2015).

42. Segers, T. \& Versluis, M. Acoustic bubble sorting for ultrasound contrast agent enrichment. Lab Chip 14, 17051714 (2014).

43. Goertz, D. E., de Jong, N. \& van der Steen, A. F. W. Attenuation and size distribution measurements of definity (TM) and manipulated definity (TM) populations. Ultrasound Med. Biol. 33, 1376-1388 (2007).

44. Feshitan, J. A., Chen, C. C., Kwan, J. J. \& Borden, M. A. Microbubble size isolation by differential centrifugation. J. Colloid Interface Sci. 329, 316-324 (2009).

45. Segers, T. et al. Monodisperse Versus Polydisperse Ultrasound Contrast Agents: Non-Linear Response, Sensitivity, and Deep Tissue Imaging Potential. Ultrasound Med. Biol. 44, 1482-1492 (2018).

46. Segers, T., Lohse, D., Versluis, M. \& Frinking, P. Universal Equations for the Coalescence Probability and Long-Term Size Stability of Phospholipid-Coated Monodisperse Microbubbles Formed by Flow Focusing. Langmuir 33, 1032910339 (2017).

47. Hettiarachchi, K., Talu, E., Longo, M. L., Dayton, P. A. \& Lee, A. P. On-chip generation of microbubbles as a practical technology for manufacturing contrast agents for ultrasonic imaging. Lab Chip 7, 463-468 (2007).

48. Borden, M. A. et al. Influence of lipid shell physicochemical properties on ultrasound-induced microbubble destruction. IEEE Trans. Ultrason. Ferroelectr. Freq. Control 52, 1992-2002 (2005).

49. Crum, L. A. Acoustic cavitation series: part five rectified diffusion. Ultrasonics 22, 215-223 (1984).

50. Omta, R. Oscillations of a cloud of bubbles of small and not so small amplitude. J. Acoust. Soc. Am. 82, 1018-1033 (1987).

51. van der Meer, S. M. et al. Microbubble spectroscopy of ultrasound contrast agents. J. Acoust. Soc. Am. 121, 648656 (2007).

52. Kooiman, K. et al. DSPC or DPPC as main shell component influences ligand distribution and binding area of lipid- 
coated targeted microbubbles. Eur. J. Lipid Sci. Technol. 116, 1217-1227 (2014).

53. Sijl, J. et al. "Compression-only" behavior: A second-order nonlinear response of ultrasound contrast agent microbubbles. J. Acoust. Soc. Am. 129, 1729-1739 (2011).

54. Sijl, J. et al. Subharmonic behavior of phospholipid-coated ultrasound contrast agent microbubbles. J. Acoust. Soc. Am. 128, 3239-3252 (2010).

55. Hosny, N. A. et al. Mapping microbubble viscosity using fluorescence lifetime imaging of molecular rotors. Proc. Natl. Acad. Sci. 110, 9225-9230 (2013).

56. de Jong, N. et al. 'Compression-Only' Behavior of Phospholipid-Coated Contrast Bubbles. Ultrasound Med. Biol. 33, 653-656 (2007).

57. Luan, Y. et al. Acoustical Properties of Individual Liposome-Loaded Microbubbles. Ultrasound Med. Biol. 38, 21742185 (2012).

58. Kooiman, K. et al. Focal areas of increased lipid concentration on the coating of microbubbles during short toneburst ultrasound insonification. PLoS One 12, (2017).

59. Baoukina, S., Monticelli, L., Risselada, H. J., Marrink, S. J. \& Tieleman, D. P. The molecular mechanism of lipid monolayer collapse. Proc. Natl. Acad. Sci. 105, 10803-10808 (2008).

60. Versluis, M. et al. Microbubble shape oscillations excited through ultrasonic parametric driving. Phys. Rev. E - Stat. Nonlinear, Soft Matter Phys. 82, (2010).

61. Dollet, B. et al. Nonspherical Oscillations of Ultrasound Contrast Agent Microbubbles. Ultrasound Med. Biol. 34, 1465-1473 (2008).

62. Marmottant, P., Versluis, M., De Jong, N., Hilgenfeldt, S. \& Lohse, D. High-speed imaging of an ultrasound-driven bubble in contact with a wall: 'narcissus' effect and resolved acoustic streaming. Exp. Fluids 41, 147-153 (2006).

63. Vos, H. J., Dollet, B., Bosch, J. G., Versluis, M. \& de Jong, N. Nonspherical Vibrations of Microbubbles in Contact with a Wall-A Pilot Study at Low Mechanical Index. Ultrasound Med. Biol. 34, 685-688 (2008).

64. Overvelde, M. et al. Dynamics of Coated Microbubbles Adherent to a Wall. Ultrasound Med. Biol. 37, 1500-1508 (2011).

65. Helfield, B. L., Leung, B. Y. C. \& Goertz, D. E. The effect of boundary proximity on the response of individual ultrasound contrast agent microbubbles. Phys. Med. Biol. 59, 1721-1745 (2014).

66. Vos, H. J., Dollet, B., Versluis, M. \& De Jong, N. Nonspherical Shape Oscillations of Coated Microbubbles in Contact With a Wall. Ultrasound Med. Biol. 37, 935-948 (2011).

67. Luan, Y. et al. Liposome shedding from a vibrating microbubble on nanoseconds timescale. in IEEE International Ultrasonics Symposium, IUS 57-60 (2013). doi:10.1109/ULTSYM.2013.0015

68. Konofagou, E. E. Optimization of the ultrasound-induced blood-brain barrier opening. Theranostics 2, 1223-1237 (2012).

69. van Wamel, A. et al. Vibrating microbubbles poking individual cells: Drug transfer into cells via sonoporation. J. 
Control. Release 112, 149-155 (2006).

70. Prentice, P., Cuschieri, A., Dholakia, K., Prausnitz, M. \& Campbell, P. Membrane disruption by optically controlled microbubble cavitation. Nat. Phys. 1, 107-110 (2005).

71. Kooiman, K., Foppen-Harteveld, M., Der Steen, A. F. W. Van \& De Jong, N. Sonoporation of endothelial cells by vibrating targeted microbubbles. J. Control. Release 154, 35-41 (2011).

72. Luan, Y. et al. Lipid shedding from single oscillating microbubbles. Ultrasound Med. Biol. 40, 1834-1846 (2014).

73. Gelderblom, E. C. et al. Brandaris 128 ultra-high-speed imaging facility: 10 years of operation, updates, and enhanced features. in Review of Scientific Instruments 83, (2012).

74. Segers, T., de Jong, N. \& Versluis, M. Uniform scattering and attenuation of acoustically sorted ultrasound contrast agents: Modeling and experiments. J. Acoust. Soc. Am. 140, 2506-2517 (2016).

75. Cox, D. J. \& Thomas, J. L. Ultrasound-induced dissolution of lipid-coated and uncoated gas bubbles. Langmuir 26, 14774-14781 (2010).

76. Thomas, D. H. et al. The quasi-stable lipid shelled microbubble in response to consecutive ultrasound pulses. Appl. Phys. Lett. 101, (2012).

77. O'Brien, J.-P., Ovenden, N. \& Stride, E. Accounting for the stability of microbubbles to multi-pulse excitation using a lipid-shedding model. J. Acoust. Soc. Am. 130, EL180-EL185 (2011).

78. van Rooij, T. et al. Non-linear Response and Viscoelastic Properties of Lipid-Coated Microbubbles: DSPC versus DPPC. Ultrasound Med. Biol. 41, 1432-1445 (2015).

79. Fan, Z., Kumon, R. E. \& Deng, C. X. Mechanisms of microbubble-facilitated sonoporation for drug and gene delivery. Therapeutic Delivery 5, 467-486 (2014).

80. Lazarus, C., Pouliopoulos, A. N., Tinguely, M., Garbin, V. \& Choi, J. J. Clustering dynamics of microbubbles exposed to low-pressure 1-MHz ultrasound. J. Acoust. Soc. Am. 142, 3135-3146 (2017).

81. Gessner, R. C., Streeter, J. E., Kothadia, R., Feingold, S. \& Dayton, P. A. An In Vivo Validation of the Application of Acoustic Radiation Force to Enhance the Diagnostic Utility of Molecular Imaging Using 3-D Ultrasound. Ultrasound Med. Biol. 38, 651-660 (2012).

82. Dayton, P., Klibanov, A., Brandenburger, G. \& Ferrara, K. Acoustic radiation force in vivo: A mechanism to assist targeting of microbubbles. Ultrasound Med. Biol. 25, 1195-1201 (1999).

83. Frinking, P. J. A. et al. Effects of Acoustic Radiation Force on the Binding Efficiency of BR55, a VEGFR2-Specific Ultrasound Contrast Agent. Ultrasound Med. Biol. 38, 1460-1469 (2012).

84. Kaya, M. et al. Acoustic Radiation Force for Vascular Cell Therapy: In Vitro Validation. Ultrasound Med. Biol. 38, 1989-1997 (2012).

85. Lum, A. F. H. et al. Ultrasound radiation force enables targeted deposition of model drug carriers loaded on microbubbles. J. Control. Release 111, 128-134 (2006).

86. Shortencarier, M. J. et al. A method for radiation-force localized drug delivery using gas-filled lipospheres. IEEE 
Trans. Ultrason. Ferroelectr. Freq. Control 51, 822-831 (2004).

87. Palanchon, P., Tortoli, P., Bouakaz, A., Versluis, M. \& De Jong, N. Optical observations of acoustical radiation force effects on individual air bubbles. IEEE Trans. Ultrason. Ferroelectr. Freq. Control 52, 104-110 (2005).

88. Tzu-Yin, W., Wilson, K. E., Machtaler, S. \& Willmann, J. K. Ultrasound and microbubble guided drug delivery: mechanistic understanding and clinical implications. Curr. Pharm. Biotechnol. 14, 743-52 (2013).

89. Aired, L., Doinikov, A. A. \& Bouakaz, A. Effect of an elastic wall on the dynamics of an encapsulated microbubble: A simulation study. Ultrasonics 53, 23-28 (2013).

90. Garbin, V. et al. Changes in microbubble dynamics near a boundary revealed by combined optical micromanipulation and high-speed imaging. Appl. Phys. Lett. 90, (2007).

91. Marmottant, P. \& Hilgenfeldt, S. Controlled vesicle deformation and lysis by single oscillating bubbles. Nature 423, 153-156 (2003).

92. De Cock, I., Lajoinie, G., Versluis, M., De Smedt, S. C. \& Lentacker, I. Sonoprinting and the importance of microbubble loading for the ultrasound mediated cellular delivery of nanoparticles. Biomaterials 83, 294-307 (2016).

93. Kooiman, K., Emmer, M., Foppen-Harteveld, M., Van Wamel, A. \& De Jong, N. Increasing the endothelial layer permeability through ultrasound-activated microbubbles. IEEE Trans. Biomed. Eng. 57, 29-32 (2010).

94. Zhou, Y., Yang, K., Cui, J., Ye, J. Y. \& Deng, C. X. Controlled permeation of cell membrane by single bubble acoustic cavitation. J. Control. Release 157, 103-111 (2012).

95. Haige, L., Chen, C. \& Wang, D. Low-frequency ultrasound and microbubbles combined with simvastatin promote the apoptosis of MCF-7 cells by affecting the LATS1/YAP/RHAMM pathway. Mol. Med. Rep. 18, 2724-2732 (2018).

96. Cui, K. et al. Ultrasound Microbubble-Mediated Delivery of Integrin-Linked Kinase Gene Improves Endothelial Progenitor Cells Dysfunction in Pre-Eclampsia. DNA Cell Biol. 33, 301-310 (2014).

97. Kovacs, Z. I. et al. Disrupting the blood-brain barrier by focused ultrasound induces sterile inflammation. Proc. Natl. Acad. Sci. 114, E75-E84 (2017).

98. Yu, H. \& Xu, L. Cell experimental studies on sonoporation: State of the art and remaining problems. J. Control. Release 174, 151-160 (2014).

99. van Rooij, T. et al. Viability of endothelial cells after ultrasound-mediated sonoporation: Influence of targeting, oscillation, and displacement of microbubbles. J. Control. Release 238, (2016).

100. Fan, Z., Kumon, R. E. \& Deng, C. X. Mechanisms of microbubble-facilitated sonoporation for drug and gene delivery. Ther. Deliv. 5, (2014).

101. Kudo, N., Okada, K. \& Yamamoto, K. Sonoporation by single-shot pulsed ultrasound with microbubbles adjacent to cells. Biophys. J. 96, 4866-4876 (2009).

102. van Rooij, T. et al. Viability of endothelial cells after ultrasound-mediated sonoporation: Influence of targeting, oscillation, and displacement of microbubbles. J. Control. Release 238, 197-211 (2016). 
103. Qin, P., Han, T., Yu, A. C. H. \& Xu, L. Mechanistic understanding the bioeffects of ultrasound-driven microbubbles to enhance macromolecule delivery. Journal of Controlled Release 272, (2018).

104. Kaddur, K. et al. Transient transmembrane release of green fluorescent proteins with sonoporation. IEEE Trans. Ultrason. Ferroelectr. Freq. Control 57, 1558-1567 (2010).

105. Qin, P. et al. Sonoporation-Induced Depolarization of Plasma Membrane Potential: Analysis of Heterogeneous Impact. Ultrasound Med. Biol. 40, 979-989 (2014).

106. Fan, Z., Liu, H., Mayer, M. \& Deng, C. C. X. Spatiotemporally controlled single cell sonoporation. Proc. Natl. Acad. Sci. U. S. A. 109, 16486-16491 (2012).

107. Fan, Z., Kumon, R. E., Park, J. \& Deng, C. X. Intracellular delivery and calcium transients generated in sonoporation facilitated by microbubbles. J. Control. Release 142, 31-39 (2010).

108. Kumon, R. E. et al. Spatiotemporal effects of sonoporation measured by real-time calcium imaging. Ultrasound Med. Biol. 35, 494-506 (2009).

109. Park, J., Fan, Z. \& Deng, C. X. Effects of shear stress cultivation on cell membrane disruption and intracellular calcium concentration in sonoporation of endothelial cells. J. Biomech. 44, 164-169 (2011).

110. Chen, X., Wan, J. M. F. \& Yu, A. C. H. Sonoporation as a Cellular Stress: Induction of Morphological Repression and Developmental Delays. Ultrasound Med. Biol. 39, 1075-1086 (2013).

111. Leow, R. S., Wan, J. M. F. \& Yu, A. C. H. Membrane blebbing as a recovery manoeuvre in site-specific sonoporation mediated by targeted microbubbles. J. R. Soc. Interface 12, (2015).

112. Wang, M. et al. Sonoporation-induced cell membrane permeabilization and cytoskeleton disassembly at varied acoustic and microbubble-cell parameters. Sci. Rep. 8, (2018).

113. Zhong, W., Sit, W. H., Wan, J. M. F. \& Yu, A. C. H. Sonoporation induces apoptosis and cell cycle arrest in human promyelocytic leukemia cells. Ultrasound Med. Biol. 37, 2149-59 (2011).

114. Yudina, A., Lepetit-Coiffé, M. \& Moonen, C. T. W. Evaluation of the temporal window for drug delivery following ultrasound-mediated membrane permeability enhancement. Mol. Imaging Biol. 13, 239-249 (2011).

115. Derieppe, M. et al. Recruitment of endocytosis in sonopermeabilization-mediated drug delivery: A real-time study. Phys. Biol. 12, (2015).

116. Burke, C. W., Alexander, E., Timbie, K., Kilbanov, A. L. \& Price, R. J. Ultrasound-activated agents comprised of 5FUbearing nanoparticles bonded to microbubbles inhibit solid tumor growth and improve survival. Mol. Ther. 22, 321-328 (2014).

117. Qin, P., Han, T., Yu, A. C. H. \& Xu, L. Mechanistic understanding the bioeffects of ultrasound-driven microbubbles to enhance macromolecule delivery. Journal of Controlled Release 272, 169-181 (2018).

118. Zhang, Z. et al. Low Intensity Ultrasound Promotes the Sensitivity of Rat Brain Glioma to Doxorubicin by DownRegulating the Expressions of P-Glucoprotein and Multidrug Resistance Protein 1 In Vitro and In Vivo. PLoS One 8, (2013).

119. Wan, C. P. L., Jackson, J. K., Pirmoradi, F. N., Chiao, M. \& Burt, H. M. Increased Accumulation and Retention of 
Micellar Paclitaxel in Drug-Sensitive and P-Glycoprotein-Expressing Cell Lines Following Ultrasound Exposure. Ultrasound Med. Biol. 38, 736-744 (2012).

120. Meijering, B. D. M. et al. Ultrasound and microbubble-targeted delivery of macromolecules is regulated by induction of endocytosis and pore formation. Circ. Res. 104, 679-687 (2009).

121. De Cock, I. et al. Ultrasound and microbubble mediated drug delivery: acoustic pressure as determinant for uptake via membrane pores or endocytosis. J. Control. Release 197, 20-28 (2015).

122. Fekri, F., Delos Santos, R. C., Karshafian, R. \& Antonescu, C. N. Ultrasound microbubble treatment enhances clathrin-mediated endocytosis and fluid-phase uptake through distinct mechanisms. PLoS One 11, (2016).

123. Zeghimi, A., Escoffre, J. M. \& Bouakaz, A. Role of endocytosis in sonoporation-mediated membrane permeabilization and uptake of small molecules: A electron microscopy study. Phys. Biol. 12, (2015).

124. Draeger, A., Monastyrskaya, K. \& Babiychuk, E. B. Plasma membrane repair and cellular damage control: The annexin survival kit. Biochemical Pharmacology 81, 703-712 (2011).

125. Lariccia, V. et al. Massive calcium-activated endocytosis without involvement of classical endocytic proteins. J. Gen. Physiol. 137, 111-132 (2011).

126. Ibsen, S. et al. The behavior of lipid debris left on cell surfaces from microbubble based ultrasound molecular imaging. Ultrasonics 54, 2090-2098 (2014).

127. De Cock, I. Unravelling microbubble-cell interactions and drug delivery mechanisms in ultrasound-guided therapy. (2016).

128. Karshafian, R., Bevan, P. D., Williams, R., Samac, S. \& Burns, P. N. Sonoporation by Ultrasound-Activated Microbubble Contrast Agents: Effect of Acoustic Exposure Parameters on Cell Membrane Permeability and Cell Viability. Ultrasound Med. Biol. 35, 847-860 (2009).

129. Fan, Z., Chen, D. \& Deng, C. X. Improving ultrasound gene transfection efficiency by controlling ultrasound excitation of microbubbles. J. Control. Release 170, 401-413 (2013).

130. Tardoski, S. et al. Low-Intensity Ultrasound Promotes Clathrin-Dependent Endocytosis for Drug Penetration into Tumor Cells. Ultrasound Med. Biol. 41, 2740-2754 (2015).

131. Choi, J. J., Carlisle, R. C., Coviello, C., Seymour, L. \& Coussios, C. C. Non-invasive and real-time passive acoustic mapping of ultrasound-mediated drug delivery. Phys. Med. Biol. 59, 4861-4877 (2014).

132. Wei, K. et al. Interactions between microbubbles and ultrasound: In vitro and in vivo observations. J. Am. Coll. Cardiol. 29, 1081-1088 (1997).

133. Qin, P., Xu, L., Han, T., Du, L. \& Yu, A. C. H. Effect of non-acoustic parameters on heterogeneous sonoporation mediated by single-pulse ultrasound and microbubbles. Ultrason. Sonochem. 31, 107-115 (2016).

134. Gac, S. Le, Zwaan, E., Berg, A. Van Den \& Ohl, C. D. Sonoporation of suspension cells with a single cavitation bubble in a microfluidic confinement. Lab Chip 7, 1666-1672 (2007).

135. Guzmán, H. R., McNamara, A. J., Nguyen, D. X. \& Prausnitz, M. R. Bioeffects caused by changes in acoustic cavitation bubble density and cell concentration: A unified explanation based on cell-to-bubble ratio and blast 
radius. Ultrasound Med. Biol. 29, 1211-1222 (2003).

136. De Cock, l. et al. Ultrasound and microbubble mediated drug delivery: acoustic pressure as determinant for uptake via membrane pores or endocytosis. J. Control. Release 197, (2015).

137. Ferrara, K. W., Borden, M. A. \& Zhang, H. Lipid-shelled vehicles: Engineering for ultrasound molecular imaging and drug delivery. Acc. Chem. Res. 42, 881-892 (2009).

138. Xing, L. et al. Ultrasound-mediated microbubble destruction (UMMD) Facilitates the delivery of CA19-9 targeted and paclitaxel loaded mPEG-PLGA-PLL nanoparticles in pancreatic cancer. Theranostics (2016).

doi:10.7150/thno.15164

139. Kokhuis, T. J. A. et al. Secondary Bjerknes Forces Deform Targeted Microbubbles. Ultrasound Med. Biol. 39, 490506 (2013).

140. Thomas, D. H., Sboros, V., Emmer, M., Vos, H. \& Jong, N. Microbubble oscillations in capillary tubes. IEEE Trans. Ultrason. Ferroelectr. Freq. Control 60, 105-114 (2013).

141. Caskey, C. F., Kruse, D. E., Dayton, P. A., Kitano, T. K. \& Ferrara, K. W. Microbubble oscillation in tubes with diameters of 12, 25, and 195 microns. Appl. Phys. Lett. 88, 1-3 (2006).

142. Shams, M. M., Dong, M. \& Mahinpey, N. Viscosity and rheological behavior of microbubbles in capillary tubes. AIChE J. 60, 2660-2669 (2014).

143. Zheng, H. et al. Ultrasound-Driven Microbubble Oscillation and Translation Within Small Phantom Vessels. Ultrasound Med. Biol. 33, 1978-1987 (2007).

144. Helfield, B. et al. Fluid Viscosity Affects the Fragmentation and Inertial Cavitation Threshold of Lipid-Encapsulated Microbubbles. Ultrasound Med. Biol. 42, 782-794 (2016).

145. Wilhelm, S. et al. Analysis of nanoparticle delivery to tumours. Nature Reviews Materials 1, (2016).

146. Pozrikidis, C. \& Davis, J. M. Blood Flow Through Capillary Networks. in Transport in Biological Media 213-252 (2013). doi:10.1016/B978-0-12-415824-5.00006-0

147. Goertz, D. E. et al. Antitumor Effects of Combining Docetaxel (Taxotere) with the Antivascular Action of Ultrasound Stimulated Microbubbles. PLoS One 7, (2012).

148. Goertz, D. E. An overview of the influence of therapeutic ultrasound exposures on the vasculature: High intensity ultrasound and microbubble-mediated bioeffects. International Journal of Hyperthermia 31, 134-144 (2015).

149. Todorova, M. et al. Antitumor effects of combining metronomic chemotherapy with the antivascular action of ultrasound stimulated microbubbles. Int. J. Cancer 132, 2956-2966 (2013).

150. Wood, A. K. W., Schultz, S. M., Lee, W. M. F., Bunte, R. M. \& Sehgal, C. M. Antivascular ultrasound therapy extends survival of mice with implanted melanomas. Ultrasound Med. Biol. 36, 853-857 (2010).

151. Wang, J. et al. Selective depletion of tumor neovasculature by microbubble destruction with appropriate ultrasound pressure. Int. J. Cancer 137, 2478-2491 (2015). 
microbubble cavitation. Circ. Cardiovasc. Imaging 8, (2015).

153. Rix, A. et al. Influence of repetitive contrast agent injections on functional and molecular ultrasound measurements. Ultrasound Med. Biol. 40, 2468-2475 (2014).

154. Belcik, J. T. et al. Augmentation of Muscle Blood Flow by Ultrasound Cavitation Is Mediated by ATP and Purinergic Signaling. Circulation 135, 1240-1252 (2017).

155. Alemany-Ribes, M. \& Semino, C. E. Bioengineering 3D environments for cancer models. Adv. Drug Deliv. Rev. 79, 40-49 (2014).

156. Weigelt, B., Ghajar, C. M. \& Bissell, M. J. The need for complex 3D culture models to unravel novel pathways and identify accurate biomarkers in breast cancer. Adv. Drug Deliv. Rev. 69-70, 42-51 (2014).

157. Zanoni, M. et al. 3D tumor spheroid models for in vitro therapeutic screening: A systematic approach to enhance the biological relevance of data obtained. Sci. Rep. 6, (2016).

158. Ohl, C. D. et al. Sonoporation from jetting cavitation bubbles. Biophys. J. 91, 4285-4295 (2006).

159. Dimcevski, G. et al. A human clinical trial using ultrasound and microbubbles to enhance gemcitabine treatment of inoperable pancreatic cancer. J. Control. Release 243, (2016).

160. Carpentier, A. et al. Clinical trial of blood-brain barrier disruption by pulsed ultrasound. Sci. Transl. Med. 8, 343re2343re2 (2016).

161. Song, K.-H., Harvey, B. K. \& Borden, M. A. State-of-the-art of microbubble-assisted blood-brain barrier disruption. Theranostics 8, 4393-4408 (2018).

162. Lipsman, N. et al. Initial experience of blood-brain barrier opening for chemotherapeutic drug delivery to brain tumours by MR-guided focused ultrasound. Neuro. Oncol. 19, vi9-vi9 (2017).

163. McDannold, N., Arvanitis, C. D., Vykhodtseva, N. \& Livingstone, M. S. Temporary disruption of the blood-brain barrier by use of ultrasound and microbubbles: Safety and efficacy evaluation in rhesus macaques. Cancer Res. 72, 3652-3663 (2012).

164. Liu, H. L. et al. Hemorrhage Detection During Focused-Ultrasound Induced Blood-Brain-Barrier Opening by Using Susceptibility-Weighted Magnetic Resonance Imaging. Ultrasound Med. Biol. 34, 598-606 (2008).

165. McDannold, N., Vykhodtseva, N. \& Hynynen, K. Effects of Acoustic Parameters and Ultrasound Contrast Agent Dose on Focused-Ultrasound Induced Blood-Brain Barrier Disruption. Ultrasound Med. Biol. 34, 930-937 (2008).

166. Xing, L. et al. Ultrasound-mediated microbubble destruction (UMMD) Facilitates the delivery of CA19-9 targeted and paclitaxel loaded mPEG-PLGA-PLL nanoparticles in pancreatic cancer. Theranostics 6, 1573-1587 (2016).

167. Yan, F. et al. Paclitaxel-liposome-microbubble complexes as ultrasound-triggered therapeutic drug delivery carriers. J. Control. Release 166, 246-255 (2013).

168. Park, J., Zhang, Y., Vykhodtseva, N., Akula, J. D. \& McDannold, N. J. Targeted and reversible blood-retinal barrier disruption via focused ultrasound and microbubbles. PLoS One 7, (2012). 
by mri-guided focused ultrasound and microbubbles. J. Ther. Ultrasound 5, 16-17 (2016).

170. Aryal, M. et al. Effects on P-glycoprotein expression after blood-brain barrier disruption using focused ultrasound and microbubbles. PLoS One 12, (2017).

171. Åslund, A. K. O. et al. Nanoparticle delivery to the brain - By focused ultrasound and self-assembled nanoparticlestabilized microbubbles. J. Control. Release 220, 287-294 (2015).

172. Sheikov, N., McDannold, N., Vykhodtseva, N., Jolesz, F. \& Hynynen, K. Cellular mechanisms of the blood-brain barrier opening induced by ultrasound in presence of microbubbles. Ultrasound Med. Biol. 30, 979-989 (2004).

173. Aron, M. M. et al. Ultrasound-mediated blood-brain barrier disruption: Correlation with acoustic emissions. J. Acoust. Soc. Am. 141, 3460-3460 (2017).

174. Theek, B. et al. Sonoporation enhances liposome accumulation and penetration in tumors with low EPR. J. Control. Release 231, 77-85 (2016).

175. Wang, T. Y. et al. Ultrasound-guided delivery of microRNA loaded nanoparticles into cancer. J. Control. Release 203, 99-108 (2015).

176. Tinkov, S. et al. New doxorubicin-loaded phospholipid microbubbles for targeted tumor therapy: In-vivo characterization. J. Control. Release 148, 368-372 (2010).

177. Kuijpers, M. J. E. et al. Complementary roles of platelets and coagulation in thrombus formation on plaques acutely ruptured by targeted ultrasound treatment: A novel intravital model. J. Thromb. Haemost. 7, 152-161 (2009).

178. Cho, H. S. et al. Localized Down-regulation of P-glycoprotein by Focused Ultrasound and Microbubbles induced Blood-Brain Barrier Disruption in Rat Brain. Sci. Rep. 6, (2016).

179. Eggen, S. et al. Ultrasound-enhanced drug delivery in prostate cancer xenografts by nanoparticles stabilizing microbubbles. J. Control. Release 187, 39-49 (2014).

180. Burke, C. W., Hsiang, Y. H. J., Alexander IV, E., Kilbanov, A. L. \& Price, R. J. Covalently linking poly(lactic-co-glycolic acid) nanoparticles to microbubbles before intravenous injection improves their ultrasound-targeted delivery to skeletal muscle. Small 7, 1227-1235 (2011).

181. Kilroy, J. P., Klibanov, A. L., Wamhoff, B. R., Bowles, D. K. \& Hossack, J. A. Localized in Vivo Model Drug Delivery with Intravascular Ultrasound and Microbubbles. Ultrasound Med. Biol. 40, 2458-2467 (2014).

182. Sontum, P. et al. Acoustic Cluster Therapy (ACT) - A novel concept for ultrasound mediated, targeted drug delivery. Int. J. Pharm. 495, 1019-1027 (2015).

183. Åslund, A. K. O. et al. Efficient enhancement of blood-brain barrier permeability using Acoustic Cluster Therapy (ACT). Theranostics 7, 23-30 (2017).

184. Wamel, A. Van et al. Acoustic Cluster Therapy (ACT) - Pre-clinical proof of principle for local drug delivery and enhanced uptake. J. Control. Release 224, 158-164 (2016).

185. Van Wamel, A. et al. Acoustic Cluster Therapy (ACT) enhances the therapeutic efficacy of paclitaxel and Abraxane ${ }^{\circledR}$ for treatment of human prostate adenocarcinoma in mice. J. Control. Release 236, 15-21 (2016). 
186. Snipstad, S. et al. Sonopermeation to improve drug delivery to tumors: from fundamental understanding to clinical translation. Expert Opin. Drug Deliv. 15, 1249-1261 (2018).

187. Pouliopoulos, A. N. et al. Rapid short-pulse sequences enhance the spatiotemporal uniformity of acoustically driven microbubble activity during flow conditions. J. Acoust. Soc. Am. 140, 2469-2480 (2016).

188. Graham, S. M. et al. Inertial cavitation to non-invasively trigger and monitor intratumoral release of drug from intravenously delivered liposomes. J. Control. Release 178, 101-107 (2014).

189. Sun, T. et al. Closed-loop control of targeted ultrasound drug delivery across the blood-brain/tumor barriers in a rat glioma model. Proc. Natl. Acad. Sci. 114, E10281-E10290 (2017).

190. O'Reilly, M. A. \& Hynynen, K. Blood-Brain Barrier: Real-time Feedback-controlled Focused Ultrasound Disruption by Using an Acoustic Emissions-based Controller. Radiology 263, 96-106 (2012).

191. McMahon, D. \& Hynynen, K. Acute inflammatory response following increased blood-brain barrier permeability induced by focused ultrasound is dependent on microbubble dose. Theranostics 7, 3989-4000 (2017).

192. Kovacs, Z. I., Burks, S. R. \& Frank, J. A. Focused ultrasound with microbubbles induces sterile inflammatory response proportional to the blood brain barrier opening: Attention to experimental conditions. Theranostics $\mathbf{8}$, 2245-2248 (2018).

193. McMahon, D. \& Hynynen, K. Reply to Kovacs et al.: Concerning acute inflammatory response following focused ultrasound and microbubbles in the brain. Theranostics 8, 2249-2250 (2018).

194. Sorace, A. G., Warram, J. M., Umphrey, H. \& Hoyt, K. Microbubble-mediated ultrasonic techniques for improved chemotherapeutic delivery in cancer. J. Drug Target. 20, 43-54 (2012).

195. Wang, S. et al. Optical verification of microbubble response to acoustic radiation force in large vessels with in vivo results. Invest. Radiol. 50, 772-784 (2015).

196. Haworth, K. J., Bader, K. B., Rich, K. T., Holland, C. K. \& Mast, T. D. Quantitative frequency-domain passive cavitation imaging. IEEE Trans. Ultrason. Ferroelectr. Freq. Control 64, 177-191 (2017).

197. Cleveland, R. O., Sapozhnikov, O. A., Bailey, M. R. \& Crum, L. A. A dual passive cavitation detector for localized detection of lithotripsy-induced cavitation in vitro. J. Acoust. Soc. Am. 107, 1745-1758 (2000).

198. Vignon, F. et al. Microbubble cavitation imaging. IEEE Trans. Ultrason. Ferroelectr. Freq. Control 60, 661-670 (2013).

199. Gyöngy, M. \& Coussios, C.-C. Passive cavitation mapping for localization and tracking of bubble dynamics. J. Acoust. Soc. Am. 128, EL175-EL180 (2010).

200. Bazan-Peregrino, M. et al. Cavitation-enhanced delivery of a replicating oncolytic adenovirus to tumors using focused ultrasound. J. Control. Release 169, 40-47 (2013).

201. Choi, J. J. et al. Noninvasive and localized blood-brain barrier disruption using focused ultrasound can be achieved at short pulse lengths and low pulse repetition frequencies. J. Cereb. Blood Flow Metab. 31, 725-737 (2011).

202. Segers, T., Gaud, E., Versluis, M. \& Frinking, P. High-precision acoustic measurements of the nonlinear dilatational elasticity of phospholipid coated monodisperse microbubbles. Soft Matter (2018). doi:10.1039/C8SM00918J 


\section{Table of content graphic}

Fundamental microbubble behavior

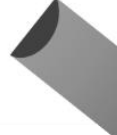

Microbubble-cell interaction

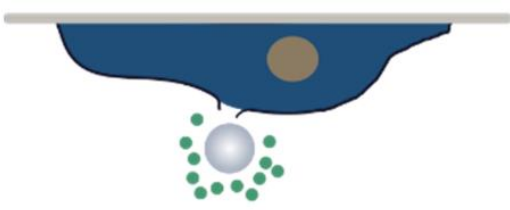

Clinical translation

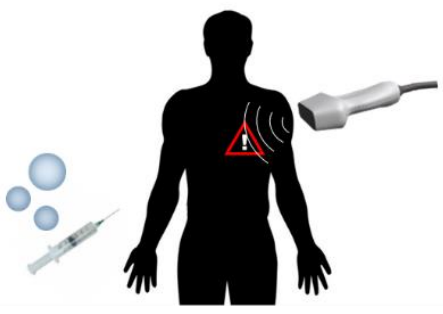

